\title{
DEVELOPMENT OF ACID-SENSITIVE PLATINUM(II) COMPLEXES WITH PROTEIN-BINDING PROPERTIES
}

\author{
M. T. Schütte ${ }^{1}$, R. Mülhaupt ${ }^{1}$, and F. Kratz ${ }^{2 *}$ \\ ' Institute of Macromolecular Chemistry, University of Freiburg, \\ Stefan-Meier-Straße 31, 79104 Freiburg, Germany \\ 2 Tumor Biology Center, Department of Medical Oncology, Clinical Research, \\ Breisacher Straße 117, 79106 Freiburg, Germany
}

\begin{abstract}
Four new protein-binding platinum(II) complexes, 10, 11, 21, 22, in which the dichloroplatinum moiety is coordinated either to a carbon-substituted or a nitrogen-substituted ethylene diamino ligand, were prepared in ten-step syntheses. According to pH-dependent stability studies with strictly related compounds, 11 and 22 exhibit acid-sensitive properties.
\end{abstract}

\section{Introduction}

Cisplatin and carboplatin are potent antineoplastic drugs which are widely used in anticancer chemotherapy. They show their best results in the treatment of testicular and ovarian carcinoma and are also effective against bladder tumours and tumours of the head and neck [1]. However, both platinum complexes do not accumulate in tumour tissues but are distributed rapidly throughout the body after parenteral administration causing several side-effects such as nephrotoxicity, myelotoxicity, ototoxicity, nausea and vomiting [1].

Hence, a major goal in anticancer chemotherapy is the development of drug delivery systems that transport the drug to the tumour while largely sparing the healthy tissues of the body. In recent years, numerous preclinical studies have shown that macromolecules such as synthetic polymers and serum proteins are taken up by tumour tissue [2]. These polymers can accumulate in solid tumours due to an enhanced vascular permeability of tumour blood vessels for circulating macromolecules combined with a lacking lymphatic drainage system in the tumour.

Following this targeting strategy, we have developed several drug protein conjugates with organic anticancer drugs, e.g. with anthracyclines or the alkylating agent chlorambucil [3-10]. In order to bind an active cis-configurated platinum(II) complex to the surface of a macromolecular carrier, we set out to design appropriate ligands that are able to form a complex with platinum(II) and are also able to bind to the respective macromolecule as shown by the general structure in Figure 1:

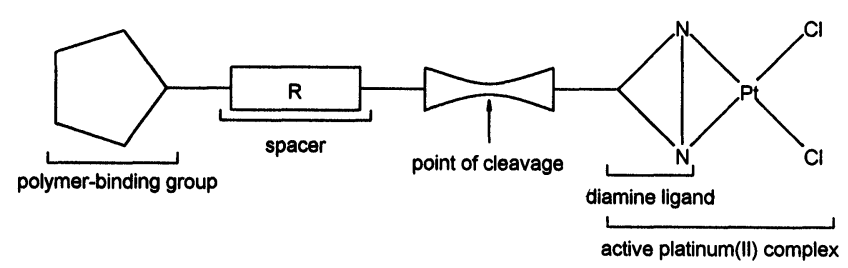

Figure 1: General structure of platinum(II) complexes capable of binding to macromolecules

In 1998, we reported on two platinum complexes in which the platinum moiety was bound to a spacer through a stable aromatic or aliphatic ester bond and also contained a maleimide group that is able to react specifically with thiolated macromolecules (see Figure 2) [11].

\footnotetext{
* Corresponding author: tel.: +49 761206 2176; fax: +49 761206 1899; e-mail· felix@tumorbio.uni-freiburg.de
} 

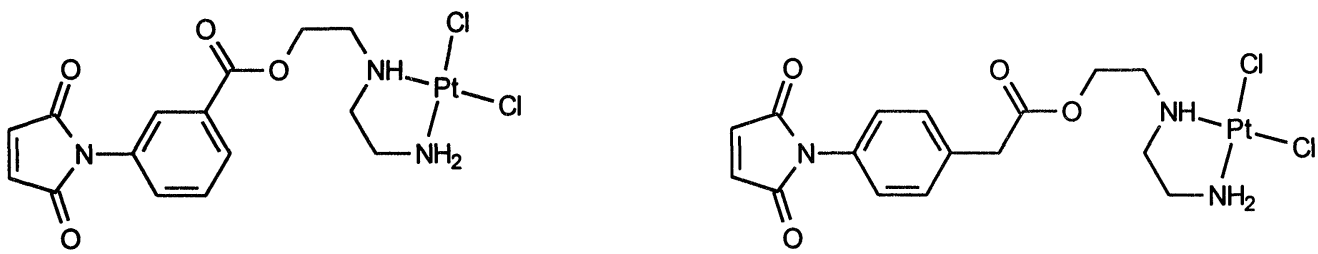

Figure 2: Structures of two platinum complexes with maleimide groups and ester bonds

However, recent studies in our group have revealed that the chemical bond between the drug and the carrier plays a crucial role for in vitro and in vivo activity. In our protein conjugates realised with anthracyclines or alkylating agents, an acid-sensitive linker was used which acts as a predetermined breaking-point releasing the protein-bound drug either in the acidic environment of tumour tissue or in the acidic endosomal or lysosomal compartments after cellular uptake of the conjugate by the tumour cell $[3,10]$.

In subsequent biological studies, we were able to establish a correlation between the acid-sensitivity of the chemical link and the antitumour activity of the conjugates: Drug protein conjugates in which the drug was bound to the protein through an acid-sensitive carboxylic hydrazone bond showed high in vitro and in vivo activity, whereas conjugates containing an ester or amide bond showed only marginal efficacy [4-7,9].

Carboxylic hydrazones have proven very useful as predetermined breaking-points because they are sufficiently stable at $\mathrm{pH} 7.4$ - thus preventing the drug from being released in the blood stream - and they also show acid-sensitive properties in a pH-range which is common in most tumour tissues and in relevant organelles of tumour cells ( $\mathrm{pH}$ range 4-6.5).

For these reasons, we prepared four maleimide containing platinum complexes with two different ligand systems that incorporate a carboxylic hydrazone bond. The synthesis and characterisation of these complexes are described in the following.

\section{Results and Discussion}

\subsection{Platinum complexes with a $\mathrm{N}$-(2-hydroxyethyl)-ethylene diamine ligand}

The method of preparing the platinum(II) complexes 10 and 11, which contain the N-(2-hydroxyethyl)ethylene diamine ligand as the complexing moiety, is depicted in scheme 1 . The starting compound 1 was synthesised as described previously [11]. In a first step, 1 was reacted with 4-formylbenzoyl chloride (2) and 4-acetylbenzoyl chloride (3), respectively. The resulting esters 4 and 5 could then be directly transformed into the carboxylic hydrazones 6 and 7 by reaction with 3-maleimidobenzoylhydrazide trifluoroacetate which was prepared in five steps according to the method of Beyer et al. [12].

In the next step, the Boc-protecting groups (Boc $=$ tert.-butoxycarbonyl) were removed. It proved best to use pure trifluoroacetic acid in the absence of water, thus preventing a cleavage of the acid-sensitive hydrazone bond. Isolation of 8 and 9 was achieved by adding diethyl ether resulting in the precipitation of the amine salts. In the last step, 8 and 9 were treated with potassium tetrachloroplatinate(II) in a mixture of DMF and water until the solutions became bright yellow. Upon addition of excess water the platinum complexes 10 and 11 precipitated as pale yellow solids which were collected by filtration and dried in vacuo.

\subsection{Platinum complexes with a 2,3-diaminopropionic acid ligand}

Two further protein-binding platinum complexes containing the 2,3-diaminopropionic acid ligand were synthesised as depicted in scheme 2. Initially, the starting material, D,L-2,3-diaminopropionic acid hydrochloride, was Boc-protected by reaction with di-tert.-butyldicarbonate in a methanol/ $\mathrm{KOH} / \mathrm{water}$ mixture. The resulting carboxylic acid 12 was then reacted with 4-hydroxybenzaldehyde (13) and 4hydroxyacetophenone (14), respectively, to form the esters 15 and 16.

The next reaction steps were performed in analogy to those described for the platinum complexes 10 and 11 . In the last step, the diamine trifluoroacetates were dissolved in DMF/water and neutralised with diluted $\mathrm{KOH}$. Subsequently, potassium tetrachloroplatinate(II) was added, and the solutions were stirred at room temperature until the colour changed to yellow. Upon addition of water, 21 and 22 were obtained as pale yellow solids. 


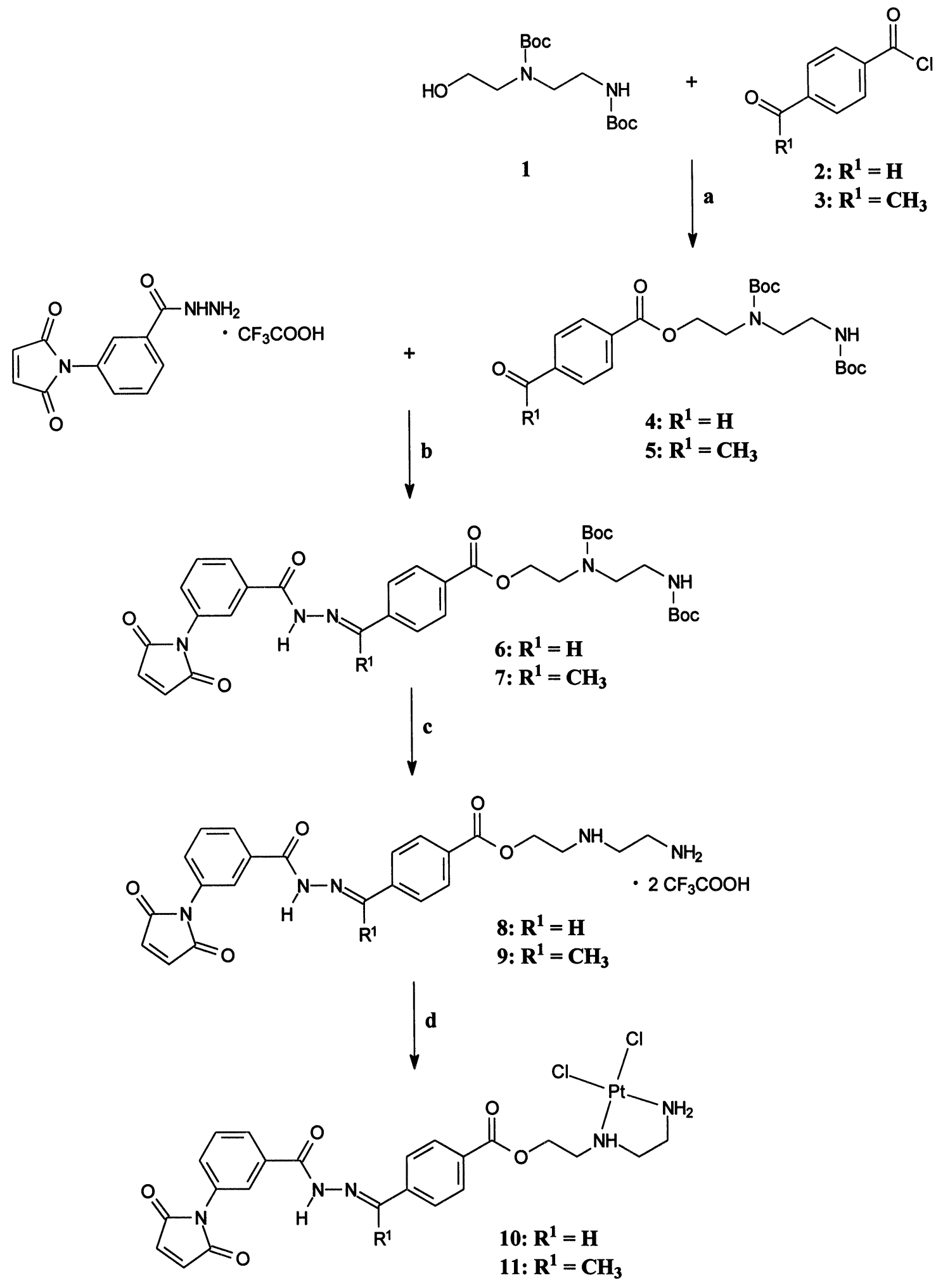

a: $\mathrm{Et}_{3} \mathrm{~N}$ / THF; b: THF, c: $1 . \mathrm{CF}_{3} \mathrm{COOH}, 2 . \mathrm{Et}_{2} \mathrm{O}, \mathrm{d}: \mathrm{K}_{2} \mathrm{PtCl}_{4}, \mathrm{DMF} / \mathrm{H}_{2} \mathrm{O}$

Scheme 1 
<smiles>CC(C)CNC(CNC(=O)OC(C)(C)C)C(=O)O</smiles>

12<smiles>[R]C(=O)c1ccc(O)cc1</smiles><smiles>[Y19][As][R4]([H])=C</smiles><smiles>NNC(=O)c1cccc(N2C(=O)C=CC2=O)c1</smiles>

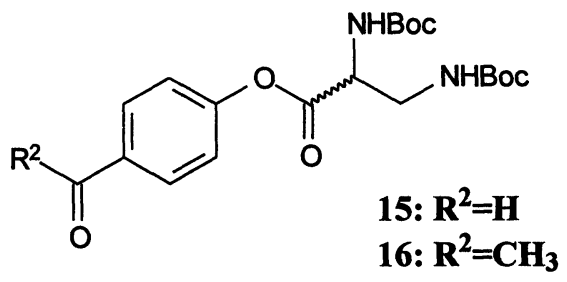<smiles>[V]C1CCCC1</smiles>

16: $\mathrm{R}^{2}=\mathrm{CH}_{3}$

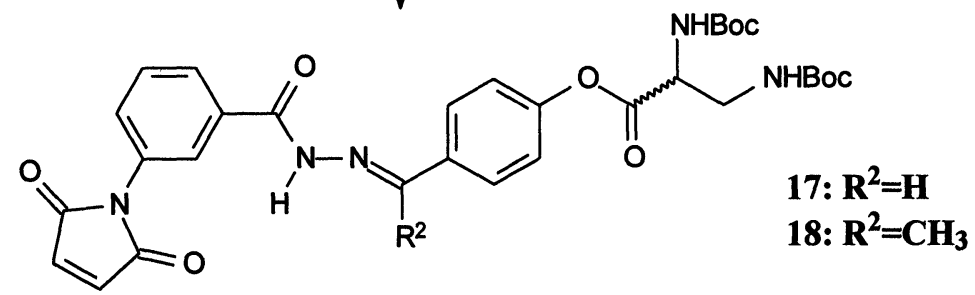

d<smiles>[R]/C(=N\N(N)C(=O)c1cccc(N2C(=O)C=CC2=O)c1)c1ccc(OC(=O)[C@H](N)CN)cc1</smiles>

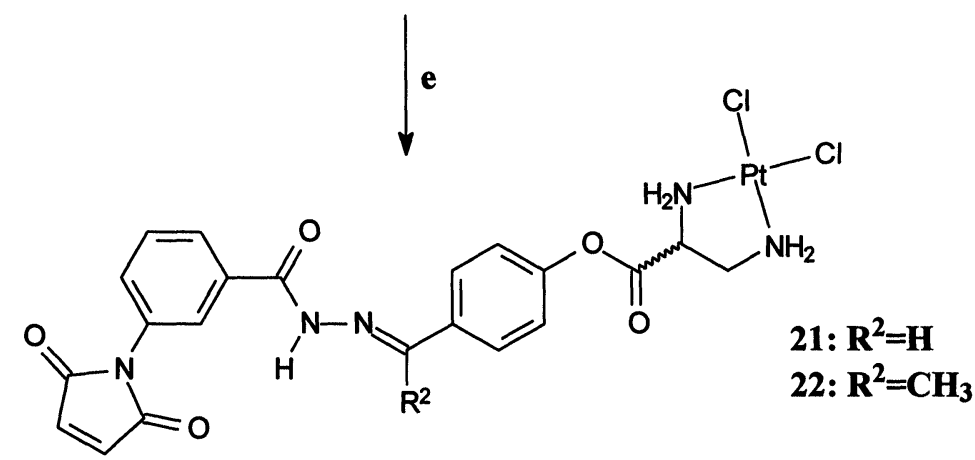

a: $(\mathrm{Boc})_{2} \mathrm{O}, \mathrm{MeOH} / \mathrm{KOH} / \mathrm{H}_{2} \mathrm{O}$; b: DCC, DMAP, THF; c: THF; d: 1. $\mathrm{CF}_{3} \mathrm{COOH}, 2$. $\mathrm{Et}_{2} \mathrm{O}$; e: $\mathrm{K}_{2} \mathrm{PtCl}_{4}, \mathrm{DMF} / \mathrm{H}_{2} \mathrm{O}$

Scheme 2 


\subsection{Characterisation}

All synthesised compounds were characterised through ${ }^{1} \mathrm{H}$ and ${ }^{13} \mathrm{C}$ NMR spectroscopy and additionally by elemental analysis (see Experimental Section). Furthermore, mass spectra of the four platinum complexes were recorded.

NMR spectroscopy of the platinum complexes 10, 11, 21 and 22 revealed the characteristic singlet peaks of the introduced maleimide group at $7.2 \mathrm{ppm}$ for the protons of the double bond, the singlets at $8.5-8.6 \mathrm{ppm}$ for the aldehyde hydrazone protons of $\mathbf{1 0}$ and $\mathbf{2 1}$ as well as the singlets at $2.4 \mathrm{ppm}$ for the methyl groups of 11 and 22. Additionally, all four complexes showed the typical signals of the maleimide group in the ${ }^{13} \mathrm{C} N M R$ spectra at 134-135 ppm for the carbon atoms of the double bond and at 169-170 ppm for the carbonyl group.

In the ${ }^{1} \mathrm{H}$ NMR and ${ }^{13} \mathrm{C}$ NMR spectra of the platinum complexes as well as of the carboxylic hydrazone compounds (6-9 and 17-20) one set of signals was observed in DMSO- $\mathrm{d}_{6}$ indicating the presence of only one stereoisomer. From a steric point of view, E-isomers are favoured, and we therefore tentatively propose that this stereoisomer is present which is in accordance with studies on simple hydrazones [13].

The ESI mass spectra of the four complexes reveal the characteristic molecular peaks $\left(\mathrm{M}^{+}+1\right)$ at 716,730 , 688 and 702 mass units for 10, 11, 21 and 22 . In addition, the peaks $\mathrm{M}^{+}+1-\mathrm{Cl}$ and $\mathrm{M}^{+}+1-2 \mathrm{Cl}$ are distinctly visible which correspond to the successive loss of the coordinatively bound chlorides. As an example, the mass spectrum of 21 in the range of 600 to 700 mass units is shown in Figure 3.

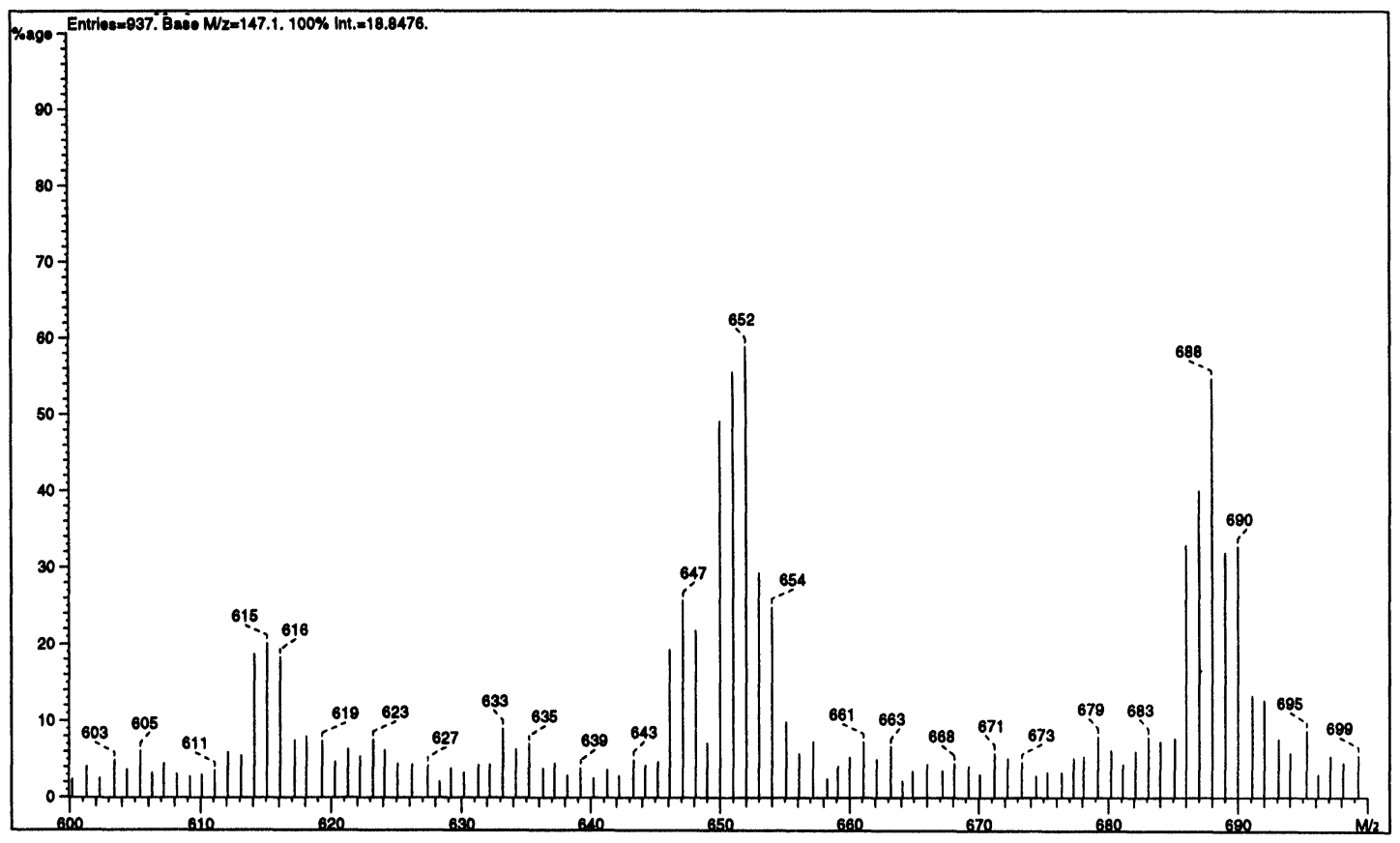

Figure 3: Mass spectrum of the platinum complex 21 in the range of 600-700 mass units

\section{3. pH-dependent hydrolysis studies}

As mentioned above, the acid-sensitivity of the link between the drug and the carrier is an important parameter for the antitumour activity of the conjugates. Hence, we wanted to perform pH-dependent hydrolysis studies to determine the acid-sensitivity of our synthesised complexes. Unfortunately, the platinum complexes 10, 11, 21 and 22 could not be used for this purpose due to their poor water-solubility. The precursors of the platinum complexes are not suited either because the maleimide group is not sufficiently stable in aqueous solutions over a period of several hours which is desired for performing hydrolysis studies.

Therefore, we synthesised the model compounds 27 and 28 which are strictly related to 17 and 18, but contain a non-hydrolysable succinic imide group instead of the maleimide group. The synthesis of the model compounds is depicted in scheme 3. 
<smiles>CC(C)C(C)C</smiles><smiles>CC#CCCCCCCCNNC(=O)c1cccc(N2C(=O)CCC2=O)c1</smiles>

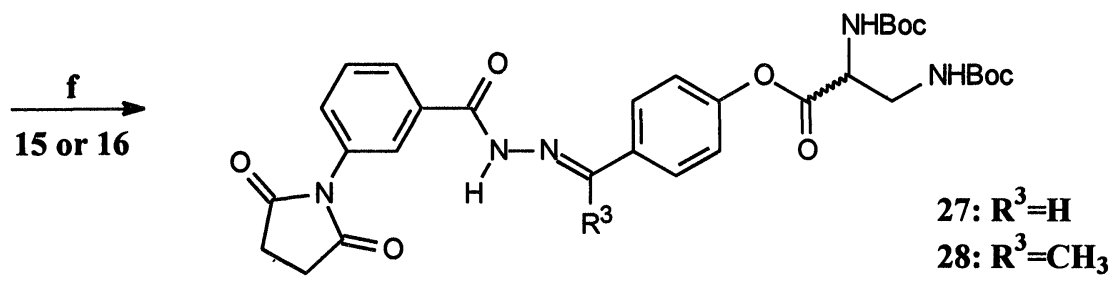

a: THF; b: $\mathrm{Ac}_{2} \mathrm{O}, \mathrm{NaOAc}$; $\mathrm{SOCl}_{2}$, toluene; d: $\mathrm{H}_{2} \mathrm{NNHBoc}$, THF; e: $1 . \mathrm{CF}_{3} \mathrm{COOH}, 2 . \mathrm{Et}_{2} \mathrm{O}$; f: EtOAc

Scheme 3: Synthesis of the model compounds 27 and 28

27 and 28 were not deprotected by acid cleavage because the resulting amine salts were difficult to analyse by reverse phase HPLC using simple mixtures of water and an organic solvent. In contrast, 27 and 28 showed definite elution profiles on a C18 reverse phase HPLC-column (eluent: acetonitrile/water 1:1; retention time for 27 was 7.9 minutes and 8.9 minutes for $\mathbf{2 8}$ ).

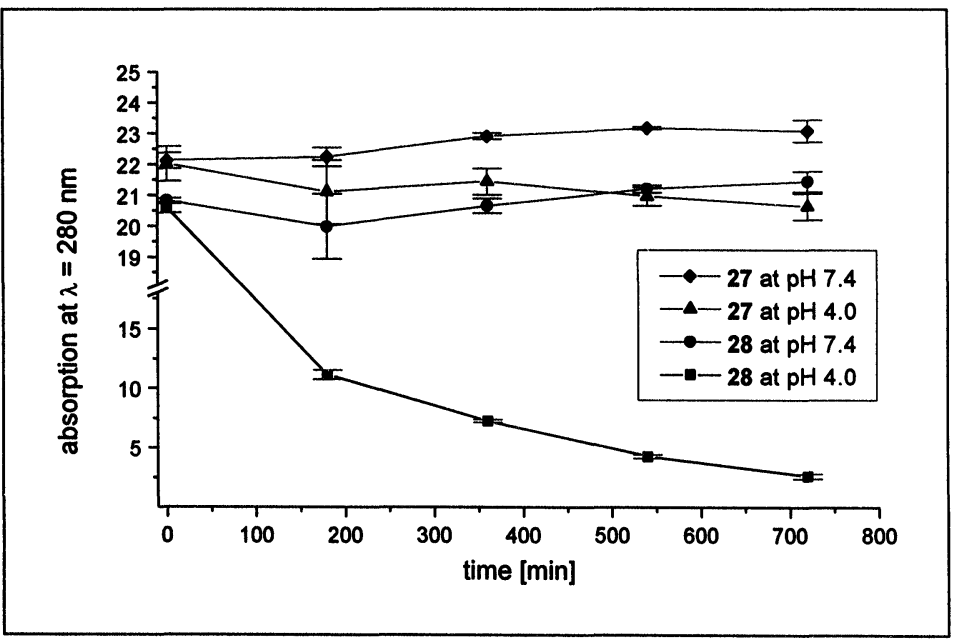

Figure 4: pH-dependent stability of the model compounds 27 and 28 at $\mathrm{pH} 7.4$ and $\mathrm{pH} 4.0$ over a range of 12 hours.

Hydrolysis studies were carried out as follows: Stock solutions of 27 and $28\left(\mathrm{c}=6 \cdot 10^{-5} \mathrm{~mol} / \mathrm{l}\right)$ in acetonitrile were prepared. $520 \mu \mathrm{l}$ of the respective stock solution were diluted with $780 \mu \mathrm{l}$ of buffer A (0.004 M sodium 
phosphate, $0.15 \mathrm{M}$ sodium chloride, $\mathrm{pH}$ 7.4) or buffer $\mathrm{B}(0.004 \mathrm{M}$ sodium phosphate, $0.15 \mathrm{M}$ sodium chloride, $\mathrm{pH} 4.0$ ). Subsequently, $50 \mu \mathrm{l}$ samples were analysed at $\mathrm{t}=0,3,6,9$ and $12 \mathrm{~h}$ by HPLC (column: Lichrosorb RP-18 $(5 \mu \mathrm{m}), 1=30 \mathrm{~cm}$, from Merck KGaA, Darmstadt, FRG) at $\lambda=280 \mathrm{~nm}$ using an autosampler (determinations were carried out in triplicate). The rates of hydrolysis were measured by the decrease of the peak areas of $\mathbf{2 7}$ and $\mathbf{2 8}$ and are depicted in Figure 4.

Both 27 and 28 do not hydrolyse at pH 7.4. At pH 4.0, however, 28 hydrolyses rapidly with a half-life of approximately 3.5 hours. Interestingly, 27 does not exhibit pronounced acid-sensitive properties with less than $10 \%$ being hydrolysed at $\mathrm{pH} 4.0$ after 12 hours.

In summary, we have prepared four new protein-binding platinum complexes in which the dichloroplatinum moiety is coordinated either to a carbon-substituted or a nitrogen-substituted ethylene diamino ligand.

According to our $\mathrm{pH}$-dependent stability studies with the strictly related model compounds $\mathbf{2 7}$ and $\mathbf{2 8}$, the complexes containing an acetophenone benzoylhydrazone bond (11 and 22) exhibit acid-sensitive properties in contrast to the two complexes $\mathbf{1 0}$ and $\mathbf{2 1}$ that contain a benzaldehyde benzoylhydrazone bond.

Preliminary experiments have shown that all four platinum complexes can be coupled to thiol-group bearing macromolecules such as human serum albumin or polyethylene glycol-SH. Respective polymer conjugates with 10, 11, 21 and 22 will be tested for antitumour activity in order to obtain structure-activity relationships for the new platinum conjugates.

\section{Acknowledgements:}

We wish to thank the Deutsche Forschungsgemeinschaft and the Schering Research Foundation for their support.

\section{Experimental Section \\ 4.1. General}

All chemicals were purchased from Aldrich, Fluka and Merck KGaA. Melting points: Büchi $530 ;{ }^{1} \mathrm{H}$ NMR and ${ }^{13}$ C NMR: Bruker ARX 300 (internal standard: TMS); electron spray MS: Finnigan-MAT 312; elemental analysis: Perkin-Elmer elemental analyser 240; silica gel chromatography on silica gel $60(0.063-0.100 \mathrm{~mm})$ from Merck KGaA; TLC: silica coated plates $60 \mathrm{~F}_{254}$ from Merck KGaA; organic solvents: p.a. grade and a gift from BASF AG or purchased from Merck $\mathrm{KGaA}, \mathrm{FRG} ; \mathrm{K}_{2} \mathrm{PtCl}_{4}$ was kindly provided by Asta Medica, FRG.

\subsection{Synthesis}

4-formylbenzoyl chloride (2), 4-acetylbenzoyl chloride (3)

$0.1 \mathrm{~mol}$ 4-carboxybenzaldehyde or 4-carboxyacetophenone was suspended in $300 \mathrm{ml}$ toluene and treated with 0.3 mol thionyl chloride $(36 \mathrm{~g})$ under reflux (according to the method of Slotta and Kethur [14]) until the suspension cleared. Excess thionyl chloride was then removed in vacuo, the remaining solution filtered and evaporated to dryness. The yellow residue was crystallised from diethyl ether to yield $15.1 \mathrm{~g}(89.6 \mathrm{mmol}, 89.6 \%) 2$ or $12.6 \mathrm{~g}(69.1 \mathrm{mmol}, 69.1 \%) 3$ as yellow needles.

2: $\mathrm{C}_{8} \mathrm{H}_{5} \mathrm{ClO}_{2}(168.45 \mathrm{~g} / \mathrm{mol})$, m.p.: $54{ }^{\circ} \mathrm{C}$, calc.: C: $56.99 \%, \mathrm{H}: 2.97 \%, \mathrm{Cl}: 21.04 \%$, found: C: $56.71 \%, \mathrm{H}: 3.22 \%, \mathrm{Cl}: 20.97 \%,{ }^{1} \mathrm{H}$ NMR $\left(300 \mathrm{MHz}, \mathrm{CDCl}_{3}\right): \delta=10.0(\mathrm{~s}, 1 \mathrm{H}, \mathrm{CHO}), 8.1(\mathrm{~d}, 2 \mathrm{H}$, arom. $\underline{\mathrm{H}}$, $\left.{ }^{3} \mathrm{~J}_{23}={ }^{3} \mathrm{~J}_{65}=12.05 \mathrm{~Hz}\right), 7.9\left(\mathrm{~d}, 2 \mathrm{H}\right.$, arom. $\left.\mathrm{H}, \mathrm{J}_{32}={ }^{3} \mathrm{~J}_{56}=12.05 \mathrm{~Hz}\right),{ }^{13} \mathrm{C}$ NMR $\left(75.4 \mathrm{MHz}, \mathrm{CDCl} \mathrm{C}_{3}\right): \delta=189.9$ $(\underline{\mathrm{C}} \mathrm{HO}), 163.4(\underline{\mathrm{COCl}}), 135.9 / 132.6 / 127.0 / 126.6($ arom. $\underline{\mathrm{C}})$

3: $\mathrm{C}_{9} \mathrm{H}_{7} \mathrm{ClO}_{2}(182.45 \mathrm{~g} / \mathrm{mol})$, m.p.: $67^{\circ} \mathrm{C}$, calc.: C: $59.19 \%, \mathrm{H}: 3.84 \%, \mathrm{Cl}: 19.43 \%$, found: C: $59.06 \%$, H: $3.76 \%$, Cl: $19.15 \%,{ }^{H} \mathrm{H}$ NMR $\left(300 \mathrm{MHz}, \mathrm{CDCl}_{3}\right): \delta=8.2\left(\mathrm{~d}, 2 \mathrm{H}\right.$, arom. $\underline{\mathrm{H}},{ }^{3} \mathrm{~J}_{23}={ }^{3} \mathrm{~J}_{65}=$ $8.66 \mathrm{~Hz}), 8.1\left(\mathrm{~d}, 2 \mathrm{H}\right.$, arom. $\left.\underline{\mathrm{H}},{ }^{3} \mathrm{~J}_{32}={ }^{3} \mathrm{~J}_{56}=8.66 \mathrm{~Hz}\right), 2.7\left(\mathrm{~s}, 3 \mathrm{H}, \mathrm{CH}_{3}\right),{ }^{13} \mathrm{C}$ NMR $\left(75.4 \mathrm{MHz}, \mathrm{CDCl}_{3}\right)$ : $\delta=197.0\left(\mathrm{CH}_{3} \underline{\underline{C O}}\right), 167.9(\underline{\mathrm{COCl}}), 141.8 / 136.5 / 131.5 / 128.6$ (arom. $\left.\underline{\mathrm{C}}\right), 27.0\left(\underline{\mathrm{CH}_{3}}\right)$

4-[3,6-bis-(tert.-butoxycarbonyl)-3,6-diazahexyloxycarbonyl]-benzaldehyde (4), 4-[3,6-bis-(tert.-butoxycarbonyl)-3,6-diazahexyloxycarbonyl|-acetophenone (5)

$16.45 \mathrm{mmol} 1$ and $16.45 \mathrm{mmol}$ of either 2 or 3 were dissolved in $30 \mathrm{ml}$ THF and $16.45 \mathrm{mmol}$ pyridine $(1.3 \mathrm{~g})$ added dropwise under vigorous stirring at room temperature. After $12 \mathrm{~h}$ pyridine hydrochloride was filtered off and the filtrate evaporated to dryness in vacuo. The crude product was dissolved in a minimal amount of ethyl acetate and purified by column chromatography (silica gel, ethyl acetate / n-hexane 1:3) to yield

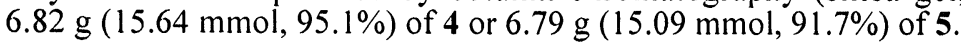

4: $\mathrm{R}_{\mathrm{f}}=0.44$ (ethyl acetate / n-hexane 1:2), $\mathrm{C}_{22} \mathrm{H}_{32} \mathrm{~N}_{2} \mathrm{O}_{7}(436 \mathrm{~g} / \mathrm{mol})$, m.p.: $84{ }^{\circ} \mathrm{C}$, calc.: C: $60.55 \%, \mathrm{H}: 7.34 \%, \mathrm{~N}: 6.42 \%$, found: $\mathrm{C}: 60.79 \%, \mathrm{H}: 7.24 \%, \mathrm{~N}: 6.46 \%,{ }^{1} \mathrm{H}$ NMR $\left(300 \mathrm{MHz}, \mathrm{CDCl}_{3}\right)$ : $\delta=10.1(\mathrm{~s}, 1 \mathrm{H}, \mathrm{C} \underline{\mathrm{HO}}), 8.2\left(\mathrm{~d}, 2 \mathrm{H}, \mathrm{H}_{\mathrm{ar}}-3 / \mathrm{H}_{\mathrm{ar}}-5,{ }^{3} \mathrm{~J}_{32}={ }^{3} \mathrm{~J}_{56}=8.32 \mathrm{~Hz}\right), 7.9\left(\mathrm{~d}, 2 \mathrm{H}, \mathrm{H}_{\mathrm{ar}}-2 / \mathrm{H}_{\mathrm{ar}}-6,{ }^{3} \mathrm{~J}_{23}={ }^{3} \mathrm{~J}_{65}=8.32\right.$ $\mathrm{Hz}), 4.9(\mathrm{~d}, 1 \mathrm{H}, \mathrm{N} \underline{\mathrm{H}}), 4.5\left(\mathrm{~s}, 2 \mathrm{H}, \mathrm{OCH}_{2}\right), 3.6 / 3.4 / 3.3\left(3 \mathrm{~s}, 6 \mathrm{H}, 3 \mathrm{NCH}_{2}\right), 1.4\left(\mathrm{~s}, 18 \mathrm{H}, 6 \mathrm{CH}_{3}\right),{ }^{13} \mathrm{C} \mathrm{NMR}$

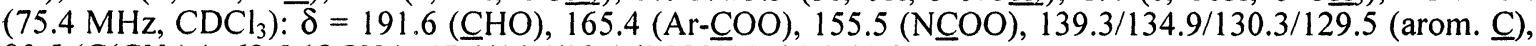
$80.5\left(\underline{\mathrm{C}}\left(\mathrm{CH}_{3}\right)_{3}\right), 63.5\left(\mathrm{OCH}_{2}\right), 47.4 / 46.6 / 39.4\left(3 \mathrm{~N}^{\mathrm{C}} \mathrm{H}_{2}\right), 28.3\left(6 \mathrm{CH}_{3}\right)$

5: $\mathrm{R}_{\mathrm{f}}=0.43$ (ethyl acetate / n-hexane 1:2), $\mathrm{C}_{23} \mathrm{H}_{34} \mathrm{~N}_{2} \mathrm{O}_{7}(450 \mathrm{~g} / \mathrm{mol})$, m.p.: $84{ }^{\circ} \mathrm{C}$, calc.: C: $61.33 \%, \mathrm{H}: 7.56 \%, \mathrm{~N}: 6.22 \%$, found: C: $61.08 \%, \mathrm{H}: 7.31 \%, \mathrm{~N}: 6.08 \%,{ }^{1} \mathrm{H}$ NMR $\left(300 \mathrm{MHz}, \mathrm{CDCl}_{3}\right)$ : 
$\delta=8.1\left(\mathrm{dd}, 2 \mathrm{H}, \mathrm{H}_{\mathrm{ar}}-3 / \mathrm{H}_{\mathrm{ar}}-5,{ }^{3} \mathrm{~J}_{32}={ }^{3} \mathrm{~J}_{56}=6.79 \mathrm{~Hz},{ }^{4} \mathrm{~J}_{35}=1.89 \mathrm{~Hz}\right), 7.9\left(\mathrm{dd}, 2 \mathrm{H}, \mathrm{H}_{\mathrm{ar}}-2 / \mathrm{H}_{\mathrm{ar}}-6,{ }^{3} \mathrm{~J}_{23}={ }^{3} \mathrm{~J}_{65}=6.79\right.$ $\left.\mathrm{Hz},{ }^{4} \mathrm{~J}_{26}=0.56 \mathrm{~Hz}\right), 4.8(\mathrm{~d}, 1 \mathrm{H}, \mathrm{NH}), 4.4\left(\mathrm{~s}, 2 \mathrm{H}, \mathrm{OCH}_{2}\right), 3.7 / 3.4 / 3.3\left(3 \mathrm{~s}, 6 \mathrm{H}, 3 \mathrm{NCH}_{2}\right), 2.6\left(\mathrm{~s}, 3 \mathrm{H}, \mathrm{COCH}_{3}\right)$ $1.3\left(\mathrm{~s}, 18 \mathrm{H}, 6 \mathrm{CH}_{3}\right),{ }^{13} \mathrm{C}$ NMR $\left(75.4 \mathrm{MHz}, \mathrm{CDCl}_{3}\right): \delta=197.5\left(\mathrm{COCH}_{3}\right), 165.5(\mathrm{Ar}-\mathrm{COO}), 155.5(\mathrm{NCOO})$,

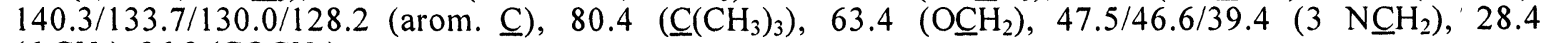
$\left(6 \mathrm{CH}_{3}\right), 26.9\left(\mathrm{COCH}_{3}\right)$

4-[3,6-bis-(tert.-butoxycarbonyl)-3,6-diazahexyloxycarbonyl|-benzaldehyde 3-maleimidobenzoylhydrazone (6), 4-[3,6-bis-(tert.-butoxycarbonyl)-3,6-diazahexyloxycarbonyl]-acetophenone 3-maleimidobenzoylhydrazone (7)

$4.59 \mathrm{mmol} 3$-maleimidobenzoylhydrazide trifluoroacetate and $4.59 \mathrm{mmol}$ of 4 or 5 were suspended in $40 \mathrm{ml}$ ethyl acetate and heated at $50^{\circ} \mathrm{C}$ until the suspension cleared. After stirring for $2 \mathrm{~h}$ at room temperature, the solvent was removed in vacuo and the crude product purified by column chromatography (silica gel, ethyl acetate / $\mathrm{n}$-hexane $1: 1)$ to yield $1.5 \mathrm{~g}(2.31 \mathrm{mmol}, 50.3 \%) 6$ or $1.3 \mathrm{~g}(1.96 \mathrm{mmol}, 42.7 \%) 7$.

6: $\mathrm{R}_{\mathrm{f}}=0.24$ (ethyl acetate / n-hexane 2:1), $\mathrm{C}_{33} \mathrm{H}_{39} \mathrm{~N}_{5} \mathrm{O}_{9}(649 \mathrm{~g} / \mathrm{mol})$, m.p.: $84{ }^{\circ} \mathrm{C}$, calc.: C: $61.02 \%, \mathrm{H}: 6.01 \%, \mathrm{~N}: 10.79 \%$, found: $\mathrm{C}: 60.62 \%, \mathrm{H}: 6.20 \%, \mathrm{~N}: 10.53 \%,{ }_{\mathrm{H}} \mathrm{NMR}\left(300 \mathrm{MHz}, \mathrm{DMSO}-\mathrm{d}_{6}\right)$ : $\delta=12.1(\mathrm{~s}, 1 \mathrm{H}, \mathrm{C}=\mathrm{NNH}), 8.5(\mathrm{~s}, 1 \mathrm{H}, \mathrm{N}=\mathrm{C} \underline{\mathrm{H}}), 8.1-7.9(\mathrm{~m}, 4 \mathrm{H}$, arom. $\underline{\mathrm{H}}), 7.7(\mathrm{~m}, 2 \mathrm{H}$, arom. $\underline{\mathrm{H}}), 7.6(\mathrm{~m}, 2 \mathrm{H}$, arom. $\underline{\mathrm{H}}), 7.2(\mathrm{~s}, 2 \mathrm{H}, \underline{\mathrm{HC}}=\mathrm{C} \underline{\mathrm{H}}), 6.8\left(\mathrm{bs}, 1 \mathrm{H}, \mathrm{CH}_{2} \mathrm{NH}\right), 4.4\left(\mathrm{~m}, 2 \mathrm{H}, \mathrm{OC}_{\mathrm{H}_{2}}\right), 3.5 / 3.3 / 3.1\left(3 \mathrm{~s}, 6 \mathrm{H}, 3 \mathrm{NC}_{2}\right), 1.3$ $\left(\mathrm{s}, 18 \mathrm{H}, 6 \mathrm{CH}_{3}\right),{ }^{13} \mathrm{C}$ NMR $\left(75.4 \mathrm{MHz}, \mathrm{DMSO}-\mathrm{d}_{6}\right): \delta=170.1(\mathrm{OC}-\mathrm{CH}=\mathrm{CH}-\mathrm{CO}), 165.5(\mathrm{Ar}-\mathrm{COO}), 162.8$ $(\mathrm{C}=\mathrm{NNH}-\mathrm{CO}), 155.9(\mathrm{C}=\mathrm{N}) 155.5(\mathrm{NCOO}), 147.1 / 139.0 / 135.2 / 132.3 / 130.9 / 130.1 / 129.5 / 127.5 / 127.1 / 126.6$ (arom. $\underline{\mathrm{C}}), 134.3(\mathrm{H} \underline{\mathrm{C}}=\underline{\mathrm{C}} \mathrm{H}) 79.2 / 78.0\left(2 \underline{\mathrm{C}}\left(\mathrm{CH}_{3}\right)_{3}\right), 63.4\left(\mathrm{OCH}_{2}\right), 47.0 / 45.8 / 38.5\left(3 \mathrm{NCH}_{2}\right), 28.4\left(6 \mathrm{CH}_{3}\right)$

7: $\mathrm{R}_{\mathrm{f}}=0.38$ (ethyl acetate / n-hexane 2:1), $\mathrm{C}_{34} \mathrm{H}_{41} \mathrm{~N}_{5} \mathrm{O}_{9}(663 \mathrm{~g} / \mathrm{mol})$, m.p.: $82^{\circ} \mathrm{C}$, calc.: C: $61.54 \%, \mathrm{H}: 6.18 \%, \mathrm{~N}: 10.56 \%$, found: C: $60.78 \%, \mathrm{H}: 6.08 \%, \mathrm{~N}: 10.04 \%,{ }_{\mathrm{H}} \mathrm{NMR}\left(300 \mathrm{MHz}, \mathrm{DMSO}-\mathrm{d}_{6}\right)$ : $\delta=10.9(\mathrm{~s}, 1 \mathrm{H}, \mathrm{C}=\mathrm{NN} \underline{\mathrm{H}}), 8.1-7.5(\mathrm{~m}, 8 \mathrm{H}$, arom. $\underline{\mathrm{H}}), 7.2(\mathrm{~s}, 2 \mathrm{H}, \underline{\mathrm{HC}}=\mathrm{CH}), 6.8\left(\mathrm{~s}, 1 \mathrm{H}, \mathrm{CH}_{2} \mathrm{NH}\right), 4.4(\mathrm{~s}, 2 \mathrm{H}$, $\left.\mathrm{OCH}_{2}\right), 3.6 / 3.4 / 3.0\left(3 \mathrm{~m}, 6 \mathrm{H}, 3 \mathrm{NCH}_{2}\right), 2.4\left(\mathrm{~N}=\mathrm{C}-\mathrm{CH}_{3}\right), 1.4\left(\mathrm{~s}, 9 \mathrm{H}, 3 \underline{\mathrm{CH}}_{3}\right), 1.3\left(\mathrm{~s}, 9 \mathrm{H}, 3 \mathrm{CH}_{3}\right){ }^{13} \mathrm{C} \mathrm{NMR}$ $\left(75.4 \mathrm{MHz}, \mathrm{DMSO}-\mathrm{d}_{6}\right): \delta=169.8(\mathrm{O} \underline{\mathrm{C}}-\mathrm{CH}=\mathrm{CH}-\mathrm{CO}), 165.2(\mathrm{Ar}-\mathrm{COO}), 163.2(\mathrm{C}=\mathrm{NNH}-\mathrm{CO}), 155.6(\underline{\mathrm{C}}=\mathrm{N})$, $154.6(\mathrm{~N} \underline{\mathrm{COO}}), 142.3 / 131.6 / 130.0 / 129.2 / 128.8 / 126.5(\operatorname{arom} . \underline{\mathrm{C}}), 134.7(\mathrm{H} \underline{\mathrm{C}}=\underline{\mathrm{CH}}) 78.8\left(\underline{\mathrm{C}}\left(\mathrm{CH}_{3}\right)_{3}\right), 59.7$ $\left(\mathrm{OCH}_{2}\right), 46.7 / 45.5 / 38.1\left(3 \mathrm{NCH}_{2}\right), 28.4\left(6 \mathrm{CH}_{3}\right), 28.0\left(\mathrm{~N}=\mathrm{C}-\mathrm{CH}_{3}\right)$

4-(3,6-diazahexyloxycarbonyl)-benzaldehyde 3-maleimidobenzoylhydrazone bis-trifluoroacetate (8), 4-(3,6-diazahexyloxycarbonyl)-acetophenone 3-maleimidobenzoylhydrazone bis-trifluoroacetate (9)

$1.54 \mathrm{mmol}$ of 6 or 7 were treated with $2 \mathrm{ml}$ trifluoroacetic acid for a few minutes at room temperature until a clear viscous yellow solution was formed. The solution was triturated with $25 \mathrm{ml}$ diethyl ether to produce 8 or 9 as precipitates, which were separated by filtration and washed twice with $25 \mathrm{ml}$ diethyl ether. The products were dried in vacuo to yield $0.93 \mathrm{~g}(1.37 \mathrm{mmol}, 89.2 \%)$ of 8 or $0.84 \mathrm{~g}(1.22 \mathrm{mmol}, 78.9 \%)$ of 9 as pale yellow powders.

8: $\mathrm{C}_{27} \mathrm{H}_{25} \mathrm{~N}_{5} \mathrm{O}_{9} \mathrm{~F}_{6}(677 \mathrm{~g} / \mathrm{mol})$, m.p.: $92{ }^{\circ} \mathrm{C}$, calc.: C: $47.86 \%, \mathrm{H}: 3.69 \%$, N: $10.34 \%$, found: $\mathrm{C}: 46.25 \%$, $\mathrm{H}: 3.71 \%, \mathrm{~N}: 9.72 \%,{ }^{1} \mathrm{H}$ NMR $\left(300 \mathrm{MHz}, \mathrm{DMSO}-\mathrm{d}_{6}\right): \delta=12.2(\mathrm{~s}, 1 \mathrm{H}, \mathrm{C}=\mathrm{NNH}), 9.3\left(\mathrm{bs}, 3 \mathrm{H}, \mathrm{NH}_{3}{ }^{+}\right), 8.5$ $(\mathrm{s}, 1 \mathrm{H}, \mathrm{N}=\mathrm{CH}), 8.2-7.7(\mathrm{~m}, 8 \mathrm{H}$, arom. $\underline{\mathrm{H}}), 7.6\left(\mathrm{~m}, 2 \mathrm{H}, \mathrm{NH}_{2}^{+}\right), 7.2(\mathrm{~s}, 2 \mathrm{H}, \underline{\mathrm{H}} \underline{\mathrm{C}}=\mathrm{C} \underline{\mathrm{H}}), 4.5\left(\mathrm{~s}, 2 \mathrm{H}, \mathrm{OC}_{\mathrm{H}}\right)$, 3.6/3.4/3.2 (3m, 6H, $\left.3 \mathrm{NCH}_{2}\right),{ }^{13} \mathrm{C}$ NMR $\left(754 \mathrm{MHz}, \mathrm{DMSO}-\mathrm{d}_{6}\right): \delta=169.7(\mathrm{OC}-\mathrm{CH}=\mathrm{CH}-\mathrm{CO}), 165.1$ (Ar$\underline{\mathrm{COO}}), 162.4(\mathrm{C}=\mathrm{NNH}-\underline{\mathrm{CO}}), 158.8\left(\mathrm{q}, \mathrm{CF}_{3} \mathrm{COOH}, J=30.2 \mathrm{~Hz}\right), 156.1(\underline{\mathrm{C}}=\mathrm{N}), 147.7 / 146.8 /$ $138.9 / 133.9 / 131.9 / 130.1 / 129.1 / 127.0 / 126.7 / 126.2($ arom. C) $), 134.7(\mathrm{H} \underline{\mathrm{C}}=\underline{\mathrm{CH}}), 116.7\left(\mathrm{q}, \underline{\mathrm{C}} \mathrm{F}_{3} \mathrm{COOH}, \mathrm{J}=\right.$ $294.1 \mathrm{~Hz}), 64.2\left(\mathrm{OCH}_{2}\right), 45.8 / 44.2 / 35.1\left(3 \mathrm{NCH}_{2}\right)$

9: $\mathrm{C}_{28} \mathrm{H}_{27} \mathrm{~N}_{5} \mathrm{O}_{9} \mathrm{~F}_{6}(691 \mathrm{~g} / \mathrm{mol})$, m.p.: $125^{\circ} \mathrm{C}$, calc.: C: $48.63 \%$, H: $3.91 \%$, N: $10.13 \%$, found: C: $45.98 \%$, H: $3.82 \%$, N: $9.71 \%$, ${ }^{1} \mathrm{H}$ NMR (300 MHz, DMSO-d $\left.{ }_{6}\right): \delta=10.9(\mathrm{~s}, 1 \mathrm{H}, \mathrm{C}=\mathrm{N}-\mathrm{N} \underline{\mathrm{H}}), 9.4\left(\mathrm{bs}, 3 \mathrm{H}, \mathrm{NH}_{3}{ }^{+}\right), 8.4-7.8$ $(\mathrm{m}, 8 \mathrm{H}$, arom. $\underline{\mathrm{H}}), 7.6\left(\mathrm{~m}_{3} 2 \mathrm{H}, \mathrm{NH}_{2}{ }^{+}\right), 7.2(\mathrm{~s}, 2 \mathrm{H}, \underline{\mathrm{HC}}=\mathrm{CH}), 4.6\left(\mathrm{~s}, 2 \mathrm{H}, \mathrm{OC}_{2}\right), 3.5 / 3.3 / 3.2(3 \mathrm{~m}, 6 \mathrm{H}$, $\left.3 \mathrm{NCH}_{2}\right), 2.4\left(\mathrm{~N}=\mathrm{C}-\mathrm{CH}_{3}\right),{ }^{13} \mathrm{C}$ NMR $\left(75.4 \mathrm{MHz}, \mathrm{DMSO}-\mathrm{d}_{6}\right): \delta=169.8(\mathrm{OC}-\mathrm{CH}=\mathrm{CH}-\mathrm{CO}), 165.2(\mathrm{Ar}-\mathrm{COO})$, $163.4(\mathrm{C}=\mathrm{NNH}-\mathrm{CO}), 158.9\left(\mathrm{q}, \mathrm{CF}_{3} \mathrm{COOH}, \mathrm{J}=33.9 \mathrm{~Hz}\right), 155.6(\mathrm{C}=\mathrm{N}) 142.7 / 140.3 / 132.8 / 131.7 / 129.9 /$ $129.7 / 128.8 / 127.2 / 126.5$ (arom. $\underline{\mathrm{C}}), 134.7(\mathrm{H} \underline{\mathrm{C}}=\underline{\mathrm{CH}}), 116.4\left(\mathrm{q}, \underline{\mathrm{C}} \mathrm{F}_{3} \mathrm{COOH}, \mathrm{J}=295.0 \mathrm{~Hz}\right), 60.6\left(\mathrm{OCH}_{2}\right)$, $45.9 / 44.2 / 35.1\left(3 \mathrm{NCH}_{2}\right), 27.2\left(\mathrm{~N}=\mathrm{C}-\mathrm{CH}_{3}\right)$

$\mathrm{N}$-|4-|3-(3-maleimidobenzoyl)-2,3-diazaprop-1-en-1-yl|-benzoyloxyethyl|-ethylenediaminodichloroplatin(II) (10), N-[4-[4-(3-maleimidobenzoyl)-3,4-diazabut-2-en-2-yl]-benzoyloxyethyl]-ethylenediaminodichloroplatin(II) (11)

$0.14 \mathrm{mmol} \mathrm{K}_{2} \mathrm{PtCl}_{4}$ were dissolved in $20 \mathrm{ml}$ of DMF/water (80:20) and treated under stirring in the dark with $0.14 \mathrm{mmol}$ of 8 or 9 dissolved in $10 \mathrm{ml} \mathrm{DMF} /$ water. The solution was stirred at room temperature until the colour changed from red to yellow. Upon addition of $40 \mathrm{ml}$ of water the platinum complexes 10 and 11 precipitated. The suspension was kept cool for $5 \mathrm{~h}$ to $4{ }^{\circ} \mathrm{C}$ to complete precipitation. The solids were filtered off and washed twice with water, then twice with THF, twice with diethyl ether and finally dried in vacuo to yield $71.5 \mathrm{mg}(0.1 \mathrm{mmol}, 71.4 \%) 10$ or $80.2 \mathrm{mg}(0.11 \mathrm{mmol}, 78.6 \%) 11$.

10: $\mathrm{C}_{23} \mathrm{H}_{23} \mathrm{~N}_{5} \mathrm{O}_{5} \mathrm{PtCl}_{2}(715 \mathrm{~g} / \mathrm{mol})$, m.p.: decomp. $>250{ }^{\circ} \mathrm{C}$, calc.: C: $38.60 \%$, H: $3.22 \%$, N: $9.79 \%$, Pt: $27.29 \%$, Cl: $9.92 \%$, found: C: $37.28 \%$, H: $3.58 \%$, N: 9.13\%, Pt: $26.79 \%, \mathrm{Cl}: 9.05 \%$, ' $\mathrm{H}$ NMR (300 MHz, DMSO-d $\left._{6}\right): \delta=12.1(\mathrm{~s}, 1 \mathrm{H}, \mathrm{C}=\mathrm{NN} \underline{\mathrm{H}}), 8.6(\mathrm{~s}, 1 \mathrm{H}, \mathrm{N}=\mathrm{C} \underline{\mathrm{H}}), 8.2-7.5(\mathrm{~m}, 8 \mathrm{H}$, arom. $\underline{\mathrm{H}}), 7.2(\mathrm{~s}, 2 \mathrm{H}, \underline{\mathrm{HC}}=\mathrm{CH})$, $4.6\left(\mathrm{~m}, 2 \mathrm{H}, \mathrm{OCH}_{2}\right),{ }^{13} \mathrm{C}$ NMR $\left(75.4 \mathrm{MHz}, \mathrm{DMSO}-\mathrm{d}_{6}\right): \delta=169.7(\mathrm{OC}-\mathrm{CH}=\mathrm{CH}-\mathrm{CO}), 165.0$ (Ar-COO), 162.3 $(\mathrm{C}=\mathrm{NNH}-\mathrm{CO}), 156.1(\underline{\mathrm{C}}=\mathrm{N}), 134.7(\mathrm{HC}=\mathrm{CH}), 61.5\left(\mathrm{OCH}_{2}\right), 54.3 / 49.6 / 35.7\left(3 \mathrm{NC}_{2}\right)$, ESI-MS $(3.4 \mathrm{kV}$, $\mathrm{DMF} / \mathrm{MeOH} 7: 1$, rel. intensity): $\mathrm{m} / \mathrm{z} 716\left(\mathrm{M}^{+}+1,14\right), 680\left(\mathrm{M}^{+}-\mathrm{Cl}, 12\right), 643\left(\mathrm{M}^{+}-2 \mathrm{Cl}, 8\right)$ 
11: $\mathrm{C}_{24} \mathrm{H}_{25} \mathrm{~N}_{5} \mathrm{O}_{5} \mathrm{PtCl}_{2}(729 \mathrm{~g} / \mathrm{mol})$, m.p.: decomp. $>250{ }^{\circ} \mathrm{C}$, calc.: C: $39.51 \%$, H: $3.43 \%$, N: 9.60\%, Pt: $26.76 \%$, Cl: $9.73 \%$, found: C: $38.24 \%, \mathrm{H}: 3.67 \%$, N: $8.92 \%$, Pt: $25.89 \%$, Cl: $9.11 \%,{ }^{1} \mathrm{H}$ NMR $(300 \mathrm{MHz}$, DMSO- $\left.\mathrm{d}_{6}\right): \delta=10.9(\mathrm{~s}, 1 \mathrm{H}, \mathrm{C}=\mathrm{N}-\mathrm{NH}), 8.4-7.8(\mathrm{~m}, 8 \mathrm{H}$, arom. $\underline{\mathrm{H}}), 7.2(\mathrm{~s}, 2 \mathrm{H}, \underline{\mathrm{H}} \mathrm{C}=\mathrm{CH}), 2.4(\mathrm{~s}, 3 \mathrm{H}$, $\left.\mathrm{N}=\mathrm{C}-\mathrm{CH}_{3}\right),{ }^{13} \mathrm{C}$ NMR $\left(75.4 \mathrm{MHz}, \mathrm{DMSO}-\mathrm{d}_{6}\right): \delta=169.8(\mathrm{OC}-\mathrm{CH}=\mathrm{CH}-\mathrm{CO}), 165.2(\mathrm{Ar}-\mathrm{COO}), 162.5$ $(\mathrm{C}=\mathrm{NNH}-\mathrm{CO}), 155.7(\underline{\mathrm{C}}=\mathrm{N}), 134.7(\mathrm{HC}=\mathrm{CH}), 62.1\left(\mathrm{OCH}_{2}\right), 56.8 / 50.2 / 37.1\left(3 \mathrm{NCH}_{2}\right)$, ESI-MS $(3.4 \mathrm{kV}$, DMF/MeOH 7:1, rel. intensity): $m / z 730\left(\mathrm{M}^{\mp}+1,18\right), 694\left(\mathrm{M}^{+}-\mathrm{Cl}, 48\right), 657\left(\mathrm{M}^{+}-2 \mathrm{Cl}, 45\right)$

$\mathrm{N}, \mathrm{N}$ '-bis-(tert.-butoxycarbonyl)-D,L-2,3-diaminopropionic acid (12)

$0.14 \mathrm{~mol}$ di-tert.-butyldicarbonate $(30.96 \mathrm{~g})$ were added to a solution of $71.14 \mathrm{mmol} \mathrm{D}, \mathrm{L}-2,3-$ diaminopropionic acid hydrochloride $(10 \mathrm{~g})$ in a mixture of $100 \mathrm{ml}$ methanol and $50 \mathrm{ml} 4 \mathrm{~N} \mathrm{KOH}$ and the reaction mixture was stirred for $12 \mathrm{~h}$ at room temperature. After removing methanol in vacuo, the aqueous solution was acidified to $\mathrm{pH} 1$ with concentrated hydrochloric acid and extracted with ethyl acetate. The organic layer was dried over sodium sulfate and concentrated in vacuo to yield $19 \mathrm{~g}(62.5 \mathrm{mmol}, 87.9 \%)$ of 12 as a white solid. $\mathrm{C}_{13} \mathrm{H}_{24} \mathrm{~N}_{2} \mathrm{O}_{6}(304 \mathrm{~g} / \mathrm{mol})$, m.p.: $145{ }^{\circ} \mathrm{C}$, calc.: C: $51.32 \%, \mathrm{H}: 7.89 \%, \mathrm{~N}: 9.21 \%$, found: C: $50.96 \%, \mathrm{H}: 8.16 \%, \mathrm{~N}: 9.58 \%{ }^{1}{ }^{\mathrm{H}} \mathrm{NMR}\left(300 \mathrm{MHz}, \mathrm{DMSO}-\mathrm{d}_{6}\right): \delta=12.3(\mathrm{bs}, 1 \mathrm{H}, \mathrm{COOH}), 6.8(\mathrm{~d}, 1 \mathrm{H}$, CHNH), $6.7\left(\mathrm{t}, 1 \mathrm{H}, \mathrm{CH}_{2} \mathrm{NH}\right) 3.9(\mathrm{q}, 1 \mathrm{H}, \mathrm{CH}), 3.2\left(\mathrm{t}, 2 \mathrm{H}, \mathrm{CH}_{2}\right), 1.4 / 1.3\left(2 \mathrm{~s}, 18 \mathrm{H}, 6 \mathrm{CH}_{3}\right),{ }^{13} \mathrm{C} \mathrm{NMR}$ $\left(75.4 \mathrm{MHz}, \mathrm{DMSO}-\mathrm{d}_{6}\right): \delta=173.1(\underline{\mathrm{COOH}}), 156.5 / 156.1(2 \mathrm{~N} \underline{\mathrm{COO}}), 79.0 / 78.8\left(2 \underline{\mathrm{C}}\left(\mathrm{CH}_{3}\right)_{3}\right), 54.6(\underline{\mathrm{CH}}), 42.0$ $\left(\mathrm{CH}_{2}\right), 29.0\left(6 \mathrm{CH}_{3}\right)$

4-[N,N'-bis-(tert.-butoxycarbonyl)-D,L-2,3-diaminopropanoyloxy]-benzaldehyde (15), 4-[N,N'-bis-(tert.butoxycarbonyl)-D,L-2,3-diaminopropanoyloxy]-acetophenone (16)

$9.87 \mathrm{mmol} 12,9.0 \mathrm{mmol}$ of 13 or 14 and $0.1 \mathrm{mmol} \mathrm{N}, \mathrm{N}$-dimethylaminopyridine (12 mg) were dissolved in $100 \mathrm{ml} \mathrm{THF}$ and $10 \mathrm{mmol} \mathrm{N}, \mathrm{N}^{\prime}$-dicyclohexylcarbodiimide $(2.1 \mathrm{~g})$, dissolved in $20 \mathrm{ml}$ THF, were then added dropwise under stirring at $4{ }^{\circ} \mathrm{C}$. The solution was stirred for $2 \mathrm{~h}$ at $4{ }^{\circ} \mathrm{C}$, then for further $12 \mathrm{~h}$ at room temperature and finally dicyclohexyl urea was filtered off. The filtrate was evaporated to dryness, dissolved in $100 \mathrm{ml}$ ethyl acetate and washed twice with $100 \mathrm{ml}$ of a saturated $\mathrm{NaHCO}_{3}$ solution and then twice with $100 \mathrm{ml}$ water. The organic layer was dried over sodium sulfate and concentrated to a volume of $50 \mathrm{ml}$. n-hexane was added until a slight turbidity remained. In the cold at $4{ }^{\circ} \mathrm{C}$, colourless crystals formed yielding $2.97 \mathrm{~g}(7.28 \mathrm{mmol}, 73.8 \%)$ of 15 or $1.80 \mathrm{~g}(4.26 \mathrm{mmol}, 43.2 \%)$ of 16 .

15: $\mathrm{C}_{20} \mathrm{H}_{28} \mathrm{~N}_{2} \mathrm{O}_{7}(408 \mathrm{~g} / \mathrm{mol})$, m.p.: $98{ }^{\circ} \mathrm{C}$, calc.: C: $58.82 \%, \mathrm{H}: 6.86 \%, \mathrm{~N}: 6.86 \%$, found: C: $58.59 \%, \mathrm{H}: 7.15 \%, \mathrm{~N}: 6.87 \%,{ }^{1} \mathrm{H}$ NMR $\left(300 \mathrm{MHz}, \mathrm{DMSO}-\mathrm{d}_{6}\right): \delta=10.0(\mathrm{~s}, 1 \mathrm{H}, \mathrm{CHO}), 8.0(\mathrm{~d}, 2 \mathrm{H}$, arom. $\underline{\mathrm{H}}, \mathrm{J}=8.67 \mathrm{~Hz}), 7.3(\mathrm{~m}, 3 \mathrm{H}, 2$ arom. $\underline{\mathrm{H}} / \mathrm{CHNH}), 7.0\left(\mathrm{t}, 1 \mathrm{H}, \mathrm{CH}_{2} \mathrm{NH}\right), 4.3(\mathrm{q}, 1 \mathrm{H}, \mathrm{C} \underline{\mathrm{H}}), 3.4\left(\mathrm{~m}, 2 \mathrm{H}, \mathrm{CH}_{2}\right)$, $1.4 / 1.3\left(2 \mathrm{~s}, 18 \mathrm{H}, 6 \mathrm{CH}_{3}\right),{ }^{13} \mathrm{C}$ NMR (75.4 MHz, DMSO-d 6 ): $\delta=192.8(\underline{\mathrm{CHO}}), 170.3(\mathrm{CHCO}), 156.5 / 156.2$ (2 NCOO), 155.9/134.9/131.9/123.4 (arom. $\underline{\mathrm{C}}), 79.7 / 79.1\left(2 \mathrm{C}\left(\mathrm{CH}_{3}\right)_{3}\right), 55.2(\underline{\mathrm{CH}}), 41.6\left(\mathrm{CH}_{2}\right), 29.0\left(6 \mathrm{CH}_{3}\right)$

16: $\mathrm{C}_{21} \mathrm{H}_{30} \mathrm{~N}_{2} \mathrm{O}_{7}(422 \mathrm{~g} / \mathrm{mol})$, m.p.: $110{ }^{\circ} \mathrm{C}$, calc.: C: $59.72 \%, \mathrm{H}: 7.11 \%$, N: $6.64 \%$, found: C: $60.40 \%, \mathrm{H}: 7.22 \%, \mathrm{~N}: 6.79 \%,{ }^{1} \mathrm{H}$ NMR $\left(300 \mathrm{MHz}, \mathrm{DMSO}-\mathrm{d}_{6}\right): \delta=8.0\left(\mathrm{~d}, 2 \mathrm{H}, \mathrm{H}_{\mathrm{ar}}-2 / \mathrm{H}_{\mathrm{ar}}-6,{ }^{3} \mathrm{~J}_{23}={ }^{3} \mathrm{~J}_{65}=\right.$ $8.67 \mathrm{~Hz}), 7.4(\mathrm{~d}, 1 \mathrm{H}, \mathrm{CHNH}), 7.3\left(\mathrm{~d}, 2 \mathrm{H}, \mathrm{H}_{\mathrm{ar}}-3 / \mathrm{H}_{\mathrm{ar}}-5,{ }^{3} \mathrm{~J}_{32}={ }^{3} \mathrm{~J}_{56}=8.67 \mathrm{~Hz}\right), 7.0\left(\mathrm{~m}, 1 \mathrm{H}, \mathrm{CH}_{2} \mathrm{~N}\right.$ ) $), 4.3(\mathrm{~m}, 1 \mathrm{H}$, $\mathrm{CH}), 3.4\left(\mathrm{~m}, 2 \mathrm{H}, \mathrm{CH}_{2}\right), 2.6\left(\mathrm{~s}, 3 \mathrm{H}, \mathrm{COCH}_{3}\right), 1.4 / 1.3\left(2 \mathrm{~s}, 18 \mathrm{H}_{1} 6 \mathrm{CH}_{3}\right),{ }^{13} \mathrm{C}$ NMR $\left(75.4 \mathrm{MHz}, \overline{\mathrm{DMSO}}-\mathrm{d}_{6}\right): \delta=$ $197.1\left(\mathrm{COCH}_{3}\right), 169.7$ (CHCO), 155.8/155.6 (2 NCOO), 154.3/134.8/130.1/122.1 (arom. $\left.\underline{\mathrm{C}}\right), 79.0 / 78.5$ (2 $\left.\mathrm{C}\left(\mathrm{CH}_{3}\right)_{3}\right), 54.5(\underline{\mathrm{C}}), 41.0\left(\mathrm{CH}_{2}\right), 28.3 / 28.2\left(6 \mathrm{CH}_{3}\right), 26.9\left(\mathrm{COCH}_{3}\right)$

4-[N,N'-bis-(tert.-butoxycarbonyl)-D,L-2,3-diaminopropanoyloxy]-benzaldehyde 3-maleimidobenzoylhydrazone (17)

$3.68 \mathrm{mmol} 3$-maleimidubenzoylhydrazide trifluoroacetate and $3.68 \mathrm{mmol} 15$ were suspended in $30 \mathrm{ml}$ ethyl acetate and heated at $50^{\circ} \mathrm{C}$ until the suspension cleared. The product precipitated in the heat, was filtered off and crystallised from methanol to yield $1.88 \mathrm{~g}(3.03 \mathrm{mmol}, 82.3 \%) 17$.

$\mathrm{C}_{31} \mathrm{H}_{35} \mathrm{~N}_{5} \mathrm{O}_{9}(621 \mathrm{~g} / \mathrm{mol})$, m.p.: $224{ }^{\circ} \mathrm{C}$, calc.: C: $59.90 \%, \mathrm{H}: 5.64 \%, \mathrm{~N}: 11.27 \%$, found: C: $60.58 \%, \mathrm{H}: 5.34 \%, \mathrm{~N}: 11.51 \%,{ }^{1} \mathrm{H}$ NMR $\left(300 \mathrm{MHz}, \mathrm{DMSO}-\mathrm{d}_{6}\right): \delta=12.0(\mathrm{~s}, 1 \mathrm{H}, \mathrm{C}=\mathrm{NNH}), 8.5(\mathrm{~s}, 1 \mathrm{H}$, $\mathrm{N}=\mathrm{CH}), 8.0-7.4(\mathrm{~m}, 8 \mathrm{H}$, arom. $\underline{\mathrm{H}}), 7.3(\mathrm{~d}, 1 \mathrm{H}, \mathrm{CHNH}), 7.2(\mathrm{~s}, 2 \mathrm{H}, \underline{\mathrm{HC}}=\mathrm{CH}), 7.0\left(\mathrm{t}, 1 \mathrm{H}, \mathrm{CH}_{2} \mathrm{NH}\right), 4.3(\mathrm{q}, 1 \mathrm{H}$, $\mathrm{C} \underline{\mathrm{H}}), 3.5\left(\mathrm{~m}, 2 \mathrm{H}, \mathrm{CH}_{2}\right), 1.4 / 1.3\left(2 \mathrm{~s}, 18 \mathrm{H}, 6 \mathrm{CH}_{3}\right),{ }^{13} \mathrm{C}$ NMR $\left(75.4 \mathrm{MHz}, \mathrm{DMSO}-\mathrm{d}_{6}\right): \delta=169.7(\mathrm{O} \underline{\mathrm{C}}-\mathrm{CH}=\mathrm{CH}-$ $\mathrm{CO}), 169.6$ (CHCO), 162.2 (C=NNH-CO), $155.5 / 155.3 \quad(2 \quad \mathrm{NCOO}), 151.6 \quad(\mathrm{C}=\overline{\mathrm{N}}) 147.1 /$ 134.1/131.9/131.8/130.0/129.0/128.2/126.6/126.1/122.1 (arom. $\underline{C}), 134.7(\mathrm{H} \underline{C}=\underline{C} H), 78.7 / 78.2\left(2 \underline{C}\left(\mathrm{CH}_{3}\right)_{3}\right)$, $54.2(\underline{\mathrm{CH}}), 40.7\left(\mathrm{CH}_{2}\right), 28.1 / 28.0\left(6 \mathrm{CH}_{3}\right)$

4-[N, N'-bis-(tert.-butoxycarbonyl)-D,L-2,3-diaminopropanoyloxy]-acetophenone 3-maleimidobenzoylhydrazone (18)

$5.92 \mathrm{mmol} 3$-maleimidobenzoylhydrazide trifluoroacetate and $5.92 \mathrm{mmol} 16$ were suspended in $30 \mathrm{ml}$ ethyl acetate and heated at $50^{\circ} \mathrm{C}$ until the suspension cleared. The mixture was then concentrated to a minimal volume and separated by column chromatography (silica gel, ethyl acetate/ $\mathrm{n}$-hexane $3: 2$ ) to yield $2.22 \mathrm{~g}$ $(3.50 \mathrm{mmol}, 59.1 \%) 18$ as a colourless powder.

$\mathrm{R}_{\mathrm{f}}=0.35$ (ethyl acetate / n-hexane $\left.2: 1\right), \mathrm{C}_{32} \mathrm{H}_{37} \mathrm{~N}_{5} \mathrm{O}_{9}(635 \mathrm{~g} / \mathrm{mol})$, m.p.: $93{ }^{\circ} \mathrm{C}$, calc.: C: $60.47 \%$, H: $5.83 \%$, $\mathrm{N}: 11.02 \%$, found: C: $60.72 \%, \mathrm{H}: 5.45 \%$, N: $11.17 \%,{ }^{1} \mathrm{H}$ NMR $(300 \mathrm{MHz}$, DMSO-d 6 ): $\delta=10.9(\mathrm{~s}, 1 \mathrm{H}$, $\mathrm{C}=\mathrm{NN} \underline{\mathrm{H}}), 8.0-7.5(\mathrm{~m}, 6 \mathrm{H}$, arom. $\underline{\mathrm{H}}), 7.4(\mathrm{~d}, 1 \mathrm{H}, \mathrm{CHNH}), 7.2(\mathrm{~m}, 4 \mathrm{H}$, arom. $\mathrm{H} / \mathrm{HC}=\mathrm{CH}), 7.1\left(\mathrm{t}, 1 \mathrm{H}, \mathrm{CH}_{2} \mathrm{NH}\right)$, $4.3(\mathrm{~m}, 1 \mathrm{H}, \mathrm{C} \underline{\mathrm{H}}), 3.4\left(\mathrm{~m}, 2 \mathrm{H}, \mathrm{CH}_{2}\right), 2.4\left(\mathrm{~s}, 3 \mathrm{H}, \mathrm{N}=\mathrm{C}-\mathrm{C}_{\underline{H}}\right), 1.4\left(\mathrm{~s}, 9 \mathrm{H}, 3 \mathrm{C}_{\mathrm{H}_{3}}\right), 1.3\left(\mathrm{~s}, 9 \mathrm{H}, 3 \mathrm{C}_{3}\right),{ }^{13} \mathrm{C} \mathrm{NMR}$ $\left(75.4 \mathrm{MHz}, \mathrm{DMSO}-\mathrm{d}_{6}\right): \delta=170.3$ (OC-CH=CH-CO), 169.7 (CHCO), 163.1 (C=NNH-CO), $155.6 / 155.3$ $(\mathrm{NCOO}), 151.4(\underline{\mathrm{C}}=\mathrm{N}), 135.7 / 131.7 / 129.7 / 128.9 / 127.6 / 127.0 / 126.3 / 121.5$ (arom. $\underline{\mathrm{C}}), 134.7(\mathrm{H} \underline{\mathrm{C}}=\underline{\mathrm{C}} \mathrm{H})$, 78.4/78.2 $\left(\underline{\mathrm{C}}\left(\mathrm{CH}_{3}\right)_{3}\right), 54.2(\underline{\mathrm{C}} \mathrm{H}), 40.8\left(\underline{\mathrm{CH}}_{2}\right), 28.1\left(6 \underline{\mathrm{CH}}_{3}\right), 28.0\left(\mathrm{~N}=\mathrm{C}-\underline{\mathrm{C}} \mathrm{H}_{3}\right)$ 
4-(D,L-2,3-diaminopropanoyloxy)-benzaldehyde 3-maleimidobenzoylhydrazone bis-trifluoroacetate (19), 4-(D,L-2,3-diaminopropanoyloxy)-acetophenone 3-maleimidobenzoylhydrazone bistrifluoroacetate (20)

$1.61 \mathrm{mmol} 17$ or 18 were treated at room temperature with $2 \mathrm{ml}$ trifluoroacetic acid for a few minutes until a clear viscous yellow solution was formed. Under vigorous stirring, $25 \mathrm{ml}$ diethyl ether were added resulting in the precipitation of $\mathbf{1 9}$ or $\mathbf{2 0}$, which were separated by filtration and washed twice with $25 \mathrm{ml}$ diethyl ether. The products were dried in vacuo to yield $0.88 \mathrm{~g}(1.36 \mathrm{mmol}, 84.5 \%) 19$ or $0.84 \mathrm{~g}(1.27 \mathrm{mmol}, 78.7 \%) 20$.

19: $\mathrm{C}_{25} \mathrm{H}_{21} \mathrm{~N}_{5} \mathrm{O}_{9} \mathrm{~F}_{6}(649 \mathrm{~g} / \mathrm{mol})$, m.p.: $66^{\circ} \mathrm{C}$, calc.: C: $46.22 \%$, H: $3.24 \%$, N: $10.79 \%$, found: C: $44.62 \%$, $\mathrm{H}: 3.21 \%, \mathrm{~N}: 9.79 \%,{ }^{1} \mathrm{H}$ NMR $\left(300 \mathrm{MHz}, \mathrm{DMSO}-\mathrm{d}_{6}\right): \delta=12.0 \cdot(\mathrm{s}, 1 \mathrm{H} \mathrm{C}=\mathrm{NNH}), 8.7\left(\mathrm{bs}, 6 \mathrm{H}^{2} 2 \mathrm{NH}_{3}{ }^{+}\right), 8.5$ $(\mathrm{s}, 1 \mathrm{H}, \mathrm{N}=\mathrm{C} \underline{\mathrm{H}}), 8.0-7.3(\mathrm{~m}, 8 \mathrm{H}$, arom. $\underline{\mathrm{H}}), 7.2(\mathrm{~s}, 2 \mathrm{H}, \underline{\mathrm{HC}}=\mathrm{CH}), 4.6(\mathrm{t}, 1 \mathrm{H}, \mathrm{CH}), 3.5\left(\mathrm{~m}, 2 \mathrm{H}, \mathrm{CH}_{2}\right),{ }^{13} \mathrm{C} \mathrm{NMR}^{-}$ $\left(75.4 \mathrm{MHz}, \mathrm{DMSO}_{\mathrm{d}}\right): \delta=170.7$ (OC-CH=CH-CO), $166.5(\mathrm{CHCO}), 163.2(\mathrm{C}=\mathrm{NNH}-\mathrm{CO}), 159.5$ $\left(\mathrm{q}, \mathrm{CF}_{3} \mathrm{COOH}, \mathrm{J}=32.7 \mathrm{~Hz}\right), 151.7(\underline{\mathrm{C}}=\mathrm{N}), 134.9(\mathrm{HC}=\underline{\mathrm{C}} \mathrm{H}), 147.9-122.9$ (arom. $\left.\underline{\mathrm{C}}\right), 117.7\left(\mathrm{q}, \underline{\mathrm{CF}}_{3} \mathrm{COOH}, \mathrm{J}=\right.$ $297.2 \mathrm{~Hz}), 51.2(\underline{\mathrm{C}} \mathrm{H}), 39.1\left(\mathrm{CH}_{2}\right)$

20: $\mathrm{C}_{26} \mathrm{H}_{23} \mathrm{~N}_{5} \mathrm{O}_{9} \mathrm{~F}_{6}(663 \mathrm{~g} / \mathrm{mol})$, m.p.: $82{ }^{\circ} \mathrm{C}$, calc.: C: $47.06 \%, \mathrm{H}: 3.47 \%$, N: $10.56 \%$, found: C: $45.89 \%$, $\mathrm{H}: 3.39 \%, \mathrm{~N}: 9.88 \%,{ }^{1} \mathrm{H}$ NMR $\left(300 \mathrm{MHz}, \mathrm{DMSO}-\mathrm{d}_{6}\right): \delta=11.0(\mathrm{~s}, 1 \mathrm{H}, \mathrm{C}=\mathrm{NNH}), 9.0\left(\mathrm{bs}, 6 \mathrm{H}, 2 \mathrm{NH}_{3}{ }^{+}\right)$, 8.0-7.3 (m, 8H, arom. $\underline{\mathrm{H}}), 7.2(\mathrm{~s}, 2 \mathrm{H}, \underline{\mathrm{HC}}=\mathrm{C} \underline{\mathrm{H}}), 4.7(\mathrm{~m}, 1 \mathrm{H}, \mathrm{C} \underline{\mathrm{H}}), 3.6\left(\mathrm{~m}, 2 \mathrm{H}, \mathrm{C} \underline{\mathrm{H}}_{2}\right), 2.4\left(\mathrm{~s}, 3 \mathrm{H}, \mathrm{N}=\mathrm{C}-\overrightarrow{\mathrm{CH}}_{3}\right)$, ${ }^{13} \mathrm{C}$ NMR (75.4 MHz, DMSO-d $\left.)_{6}\right): \delta=169.8$ (OC $\left.-\mathrm{CH}=\mathrm{CH}-\mathrm{CO}\right), 165.6(\mathrm{CHCO}), 163.4(\mathrm{C}=\mathrm{NNH}-\mathrm{CO}), 159.5$ $\left(\mathrm{q}, \mathrm{CF}_{3} \underline{\mathrm{COOH}}, \mathrm{J}=33.1 \mathrm{~Hz}\right), 150.7(\underline{\mathrm{C}}=\mathrm{N}), 134.8(\mathrm{H} \underline{\mathrm{C}}=\underline{\mathrm{C}} \mathrm{H}), 136.5-121.5$ (arom. $\left.\underline{\mathrm{C}}\right), 117.7\left(\mathrm{q}, \underline{\mathrm{CF}} \mathrm{C}_{3} \mathrm{COOH}, \mathrm{J}=\right.$ $296.9 \mathrm{~Hz}), 50.4(\underline{\mathrm{CH}}), 40.2\left(\mathrm{CH}_{2}\right), 26.7\left(\mathrm{~N}=\mathrm{C}-\mathrm{CH}_{3}\right)$

(R,S)-1-[4-[3-(3-maleimidobenzoyl)-2,3-diazaprop-1-en-1-yl]-phenoxycarbonyl]-ethylenediaminodichloroplatin(II) (21), (R,S)-1-[4-[4-(3-maleimidobenzoyl)-3,4-diazabut-2-en-2-yl]-phenoxycarbonyl]ethylenediaminodichloroplatin(II) (22)

$0.15 \mathrm{mmol} 19$ or 20 were dissolved in $10 \mathrm{ml} \mathrm{DMF} /$ water $(80: 20)$ and neutralised with $1.5 \mathrm{ml} 0.1 \mathrm{~N} \mathrm{KOH}$.

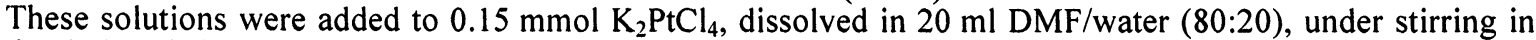
the dark. Stirring was continued until the colour changed from red to yellow. Upon addition of $40 \mathrm{ml}$ of water the platinum complexes 21 and 22 precipitated. The suspension was cooled for $5 \mathrm{~h}$ to $4{ }^{\circ} \mathrm{C}$ to complete the precipitation. The precipitate was filtered off and washed twice with water, then twice with THF, twice with diethyl ether and finally dried in vacuo to yield $88.8 \mathrm{mg}(0.13 \mathrm{mmol}, 86.7 \%) 21$ or $84.1 \mathrm{mg}(0.12 \mathrm{mmol}$, $80.0 \%) 22$.

21: $\mathrm{C}_{21} \mathrm{H}_{19} \mathrm{~N}_{5} \mathrm{O}_{5} \mathrm{PtCl}_{2}(687 \mathrm{~g} / \mathrm{mol})$, m.p.: decomp. $>250{ }^{\circ} \mathrm{C}$, calc.: C: $36.68 \%, \mathrm{H}: 2.76 \%, \mathrm{~N}: 10.19 \%$, Pt: $28.40 \%, \mathrm{Cl}: 10.32 \%$, found: $\mathrm{C}: 35.06 \%, \mathrm{H}: 2.84 \%, \mathrm{~N}: 9.77 \%, \mathrm{Pt}: 27.12 \%, \mathrm{Cl}: 9.43 \%,{ }^{1} \mathrm{H} \mathrm{NMR}$ (300 MHz, DMSO-d $\left.)_{6}\right): \delta=12.1(\mathrm{~s}, 1 \mathrm{H}, \mathrm{C}=\mathrm{N}-\mathrm{NH}), 8.5(\mathrm{~s}, 1 \mathrm{H}, \mathrm{N}=\mathrm{CH}), 8.0-7.5(\mathrm{~m}, 8 \mathrm{H}$, arom. $\mathrm{H}), 7.3(\mathrm{~d}, 2 \mathrm{H}$, $\left.\mathrm{CHN}_{2}\right), 7.2(\mathrm{~s}, 2 \mathrm{H}, \underline{\mathrm{HC}}=\mathrm{C} \underline{\mathrm{H}}), 6.8\left(\mathrm{~m}, 2 \mathrm{H}, \mathrm{CH}_{2} \underline{\mathrm{N}}_{2}\right), 4.1(\mathrm{~m}, 1 \mathrm{H}, \underline{\mathrm{CH}}), 3.1\left(\mathrm{~m}, 2 \mathrm{H}, \mathrm{CH}_{2}\right),{ }^{13} \mathrm{C} \mathrm{NMR}(75.4$ MHz, DMSO-d 6 ): $\delta=169.7$ (OC $-\mathrm{CH}=\mathrm{CH}-\underline{\mathrm{CO}}), 166.1(\mathrm{CHCO}), 162.2(\mathrm{C}=\mathrm{NNH}-\underline{\mathrm{CO}}), 148.4(\underline{\mathrm{C}}=\mathrm{N}), 134.7$ $(\mathrm{HC}=\underline{\mathrm{CH}}), 60.3(\underline{\mathrm{CH}}), 48.5\left(\underline{\mathrm{CH}}_{2}\right)$, ESI-MS $\left(3.8 \mathrm{kV}, \mathrm{DMF} / \mathrm{MeOH} 7: 1\right.$, rel. intensity): $m / z 688\left(\mathrm{M}^{+}+1,54\right)$, $652\left(\overline{\mathrm{M}}^{+}-\mathrm{Cl}, 59\right), 615\left(\mathrm{M}^{+}-2 \mathrm{Cl}, 20\right)$

22: $\mathrm{C}_{22} \mathrm{H}_{21} \mathrm{~N}_{5} \mathrm{O}_{5} \mathrm{PtCl}_{2}(701 \mathrm{~g} / \mathrm{mol})$, m.p.: decomp. $>250{ }^{\circ} \mathrm{C}$, calc.: C: $37.66 \%, \mathrm{H}: 3.00 \%, \mathrm{~N}: 9.99 \%$, Pt: $27.83 \%, \mathrm{Cl}: 10.11 \%$, found: $\mathrm{C}: 37.06 \%, \mathrm{H}: 3.57 \%, \mathrm{~N}: 10.53 \%, \mathrm{Pt}: 26.88 \%, \mathrm{Cl}: 9.42 \%,{ }^{1} \mathrm{H} \mathrm{NMR}$ $\left(300 \mathrm{MHz}, \mathrm{DMSO}-\mathrm{d}_{6}\right): \delta=10.9(\mathrm{~s}, 1 \mathrm{H}, \mathrm{C}=\mathrm{NNH}), 8.1-7.5(\mathrm{~m}, 8 \mathrm{H}$, arom. $\mathrm{H}), 7.2(\mathrm{~s}, 2 \mathrm{H}, \mathrm{HC}=\mathrm{CH}), 2.4(\mathrm{~s}, 3 \mathrm{H}$, $\left.\mathrm{N}=\mathrm{C}-\mathrm{CH}_{3}\right),{ }^{13} \mathrm{C} \mathrm{NMR}\left(75.4 \mathrm{MHz}, \mathrm{DMSO}-\mathrm{d}_{6}\right): \underline{\delta}=169.7$ (OC- $\left.\mathrm{CH}=\mathrm{CH}-\underline{\mathrm{CO}}\right), 166.7(\mathrm{CHCO}), 163.2(\mathrm{C}=\mathrm{NNH}-$ $\underline{\mathrm{CO}}), 151.8(\underline{\mathrm{C}=\mathrm{N}}), 134.7(\mathrm{H} \underline{\mathrm{C}}=\underline{\mathrm{CH}}), 60.3(\underline{\mathrm{CH}}), 48.5\left(\underline{\mathrm{CH}}_{2}\right), 26.7\left(\mathrm{~N}=\mathrm{C}-\underline{\mathrm{C}} \mathrm{H}_{3}\right), \mathrm{ESI}-\mathrm{MS}(3.4 \mathrm{kV}, \mathrm{DMF} / \mathrm{MeOH}$ $7: 1$, rel. intensity): $m / z 702\left(\bar{M}^{+}+1,45\right), 665\left(\mathrm{M}^{+}-\mathrm{Cl}, 25\right), 629\left(\mathrm{M}^{+}-2 \mathrm{Cl}, 13\right)$

3-succinimidobenzoic acid (23)

$0.36 \mathrm{~mol} \mathrm{3}$-aminobenzoic acid $(50 \mathrm{~g})$ were dissolved in $550 \mathrm{ml} \mathrm{THF}$ by heating at $50{ }^{\circ} \mathrm{C}$ and $0.43 \mathrm{~mol}$ succinic anhydride $(43 \mathrm{~g})$ were added in ten portions. After stirring for $2 \mathrm{~h}$ at room temperature the resulting precipitate was filtered off and washed with a small amount of THF. The crude product was then suspended in $100 \mathrm{ml}$ THF and treated with $1.44 \mathrm{~mol}$ acetic anhydride $(147 \mathrm{~g})$ and $0.36 \mathrm{~mol}$ sodium acetate $(30 \mathrm{~g})$ under reflux until an almost clear solution was formed. Excess acetic anhydride was hydrolysed by adding water and heating the solution under reflux for $1 \mathrm{~h}$. After cooling to room temperature, concentrated hydrochloric acid was added until 23 began to precipitate. The suspension was cooled to $4{ }^{\circ} \mathrm{C}$ for $5 \mathrm{~h}$. Then the precipitate was filtered off, washed with water and dried in vacuo to yield $71 \mathrm{~g}(0.32 \mathrm{~mol}, \mathbf{8 8 . 8 \%}) \mathbf{2 3}$.

$\mathrm{C}_{11} \mathrm{H}_{9} \mathrm{NO}_{4}(219 \mathrm{~g} / \mathrm{mol})$, m.p.: $225^{\circ} \mathrm{C}$, calc.: C: $60.27 \%$, H: $4.11 \%$, N: $6.39 \%$, found: C: $60.41 \%, \mathrm{H}: 4.10 \%$, $\mathrm{N}: 6.56 \%,{ }^{1} \mathrm{H}$ NMR $\left(300 \mathrm{MHz}, \mathrm{DMSO}_{-} \mathrm{d}_{6}\right): \delta=13.1(\mathrm{~s}, 1 \mathrm{H}, \mathrm{COOH}), 7.9(\mathrm{~m}, 2 \mathrm{H}$, arom. $\underline{\mathrm{H}}), 7.5$ (m, 2H, arom. $\underline{\mathrm{H}}), 2.7\left(\mathrm{~s}, 4 \mathrm{H}, \underline{\mathrm{H}}_{2} \mathrm{C}-\mathrm{CH}_{2}\right),{ }^{13} \mathrm{C} \mathrm{NMR}\left(75.4 \mathrm{MHz}, \mathrm{DMSO}-\mathrm{d}_{6}\right): \delta=176.8\left(\mathrm{O} \underline{\mathrm{C}}-\mathrm{CH}_{2} \mathrm{CH} \mathrm{H}_{2}-\mathrm{CO}\right)$, 166.5 (ㄷOH), 132.9/131.4/131.3/129.1/128.8/128.0 (arom. ㄷ) $), 28.4\left(\mathrm{H}_{2} \mathrm{C}-\underline{\mathrm{C}} \mathrm{H}_{2}\right)$

3-succinimidobenzoyl chloride (24)

$0.21 \mathrm{~mol} 23(46 \mathrm{~g})$ were suspended in $200 \mathrm{ml}$ of toluene and treated with 0.63 mol thionyl chloride (75 g) under reflux until a solution was formed. Excess thionyl chloride was removed by distillation and toluene was subsequently evaporated in vacuo. The brownish residue was crystallised from acetone and washed with small amounts of $\mathrm{n}$-hexane. The product was dried in vacuo to yield $38.9 \mathrm{~g}(0.16 \mathrm{~mol}, 76.2 \%)$ of 24 .

$\mathrm{C}_{11} \mathrm{H}_{8} \mathrm{NO}_{3} \mathrm{Cl}(237.45 \mathrm{~g} / \mathrm{mol})$, m.p.: $100{ }^{\circ} \mathrm{C}$, calc.: C: $55.59 \%, \mathrm{H}: 3.37 \%, \mathrm{~N}: 5.90 \%$, Cl: $14.93 \%$, found: C: $55.90 \%, \mathrm{H}: 3.19 \%, \mathrm{~N}: 5.73 \%, \mathrm{Cl}: 14.62 \%,{ }^{1} \mathrm{H}$ NMR $\left(300 \mathrm{MHz}, \mathrm{DMSO}-\mathrm{d}_{6}\right): \delta=7.9(\mathrm{~m}, 2 \mathrm{H}$, arom. $\underline{\mathrm{H}})$, 
$7.5(\mathrm{~m}, 2 \mathrm{H}$, arom. $\underline{\mathrm{H}}), 2.8\left(\mathrm{~s}, 4 \mathrm{H}, \underline{\mathrm{H}_{2}} \mathrm{C}-\mathrm{CH}_{2}\right),{ }^{13} \mathrm{C} \mathrm{NMR}\left(75.4 \mathrm{MHz}, \mathrm{DMSO}-\mathrm{d}_{6}\right): \delta=176.4\left(\mathrm{OC}_{-}-\mathrm{CH}_{2} \mathrm{CH}_{2}-\underline{\mathrm{CO}}\right)$, 166.0 ( $\underline{\mathrm{COCl}}$ ), $132.5 / 131.7 / 131.0 / 129.6 / 128.4 / 127.5$ (arom. $\underline{\mathrm{C}}), 28.5\left(\mathrm{H}_{2} \mathrm{C}-\underline{\mathrm{C}} \mathrm{H}_{2}\right)$

1-(3-succinimidobenzoyl)-2-(tert.-butoxycarbonyl)-hydrazine (25)

$42.1 \mathrm{mmol} 24(10 \mathrm{~g})$ and $42.1 \mathrm{mmol}$ tert.-butylcarbazate $(5.56 \mathrm{~g})$ were dissolved in $160 \mathrm{ml}$ THF and treated under vigorous stirring with $42.1 \mathrm{mmol}$ triethylamine $(5.85 \mathrm{ml})$. After 10 minutes the reaction was complete. The precipitated triethylammonium chloride was filtered off and the filtrate evaporated to dryness in vacuo, dissolved in ethyl acetate and washed twice with $100 \mathrm{ml} 0.5 \mathrm{~N}$ hydrochloric acid and then twice with $100 \mathrm{ml}$ saturated $\mathrm{NaCl}$ solution. The organic phase was concentrated and cooled to $0^{\circ} \mathrm{C}$ to yield $11.95 \mathrm{~g}(35.9 \mathrm{mmol}$, $85.3 \%)$ of $\mathbf{2 5}$ as a colourless powder.

$\mathrm{C}_{16} \mathrm{H}_{19} \mathrm{~N}_{3} \mathrm{O}_{5}(333 \mathrm{~g} / \mathrm{mol})$, m.p.: $184{ }^{\circ} \mathrm{C}$, calc.: C: $57.66 \%, \mathrm{H}: 5.71 \%, \mathrm{~N}: 12.61 \%$, found: $\mathrm{C}: 58.20 \%$, $\mathrm{H}: 5.94 \%, \mathrm{~N}: 12.66 \%,{ }^{1} \mathrm{H}$ NMR $\left(300 \mathrm{MHz}, \mathrm{DMSO}^{-} \mathrm{d}_{6}\right): \delta=10.3 / 8.9(2 \mathrm{~s}, 2 \mathrm{H}, 2 \mathrm{NH}), 7.9-7.4(\mathrm{~m}, 4 \mathrm{H}$, arom. $\underline{\mathrm{H}}), 2.8\left(\mathrm{~s}, 4 \mathrm{H}, \underline{\mathrm{H}}_{2} \mathrm{C}-\mathrm{CH}_{2}\right), 1.4\left(\mathrm{~s}, 9 \mathrm{H}, 3 \mathrm{CH}_{3}\right),{ }^{13} \mathrm{C}$ NMR $\left(75.4 \mathrm{MHz}\right.$, DMSO-d $\left.\mathrm{d}_{6}\right): \delta=176.7\left(\mathrm{OC}^{-\mathrm{CH}_{2} \mathrm{CH}_{2}-}\right.$ $\underline{\mathrm{CO}}$ ), 165.1 (Ar- $\underline{\mathrm{CONH}}$ ), 155.3 ( $\underline{\mathrm{COOO}}$ ), 133.2/132.9/130.3/128.9/126.5/126.4 (arom. $\underline{\mathrm{C}}), 79.2\left(\underline{\mathrm{C}}\left(\mathrm{CH}_{3}\right)_{3}\right)$, $28.4\left(3 \mathrm{CH}_{3}\right), 28.0\left(\mathrm{H}_{2} \mathrm{C}-\mathrm{CH}_{2}\right)$

3-succinimidobenzoylhydrazide trifluoroacetate (26)

$15.0 \mathrm{mmol} 25(5 \mathrm{~g})$ were treated with $20 \mathrm{ml}$ trifluoroacetic acid at room temperature, until a solution was formed. $100 \mathrm{ml}$ diethyl ether were added under vigorous stirring resulting in the precipitation of 26, which was separated by filtration and washed twice with $25 \mathrm{ml}$ diethyl ether. The product was dried in vacuo to yield $4.16 \mathrm{~g}(11.99 \mathrm{mmol}, \mathbf{7 9 . 9 \% )}$ of $\mathbf{2 6}$ as a colourless powder.

$\mathrm{C}_{13} \mathrm{H}_{12} \mathrm{~N}_{3} \mathrm{O}_{5} \mathrm{~F}_{3}(347 \mathrm{~g} / \mathrm{mol})$, m.p.: $89{ }^{\circ} \mathrm{C}$, calc.: C: $44.96 \%$, H: $3.46 \%, \mathrm{~N}: 12.10 \%$, found: C: $44.12 \%$,

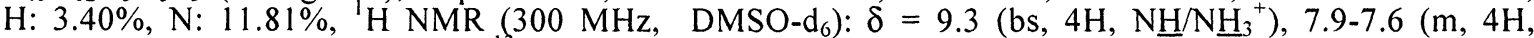
arom. $\underline{\mathrm{H}}), 2.8\left(\mathrm{~s}, 4 \mathrm{H}, \underline{\mathrm{H}}_{2} \mathrm{C}-\mathrm{CH}_{2}\right),{ }^{13} \mathrm{C}$ NMR $\left(75.4 \mathrm{MHz}, \mathrm{DMSO}-\mathrm{d}_{6}\right): \delta=177.0\left(\mathrm{OC}^{\mathrm{C}}-\mathrm{CH}_{2} \mathrm{CH}_{2}-\mathrm{CO}\right), 165.4$ $(\mathrm{Ar}-\mathrm{CONH}), 162.1\left(\mathrm{q}, \mathrm{CF}_{3} \mathrm{COOH}, \mathrm{J}=33.7 \mathrm{~Hz}\right), 136.7 / 134.9 / 134.7 / 132.8 / 130.4 / 130.0$ (arom. $\left.\underline{\mathrm{C}}\right), 121.9$ (q, $\left.\mathrm{CF}_{3} \mathrm{COOH}, \mathrm{J}=295.4 \mathrm{~Hz}\right), 28.7\left(\mathrm{H}_{2} \underline{\mathrm{C}}-\mathrm{CH}_{2}\right)$

4-[N,N'-bis-(tert.-butoxycarbonyl)-D,L-2,3-diaminopropanoyloxy|-benzaldehyde 3-succinimidobenzoylhydrazone (27)

$5.76 \mathrm{mmol} 26(2 \mathrm{~g})$ and $5.76 \mathrm{mmol} 15(2.35 \mathrm{~g})$ were suspended in $50 \mathrm{ml}$ ethyl acetate and heated at $50{ }^{\circ} \mathrm{C}$ until precipitation occurred. The precipitate was filtered off and crystallised from methanol to yield $2.29 \mathrm{~g}(3.68 \mathrm{mmol}, 63.9 \%) 27$.

$\mathrm{C}_{31} \mathrm{H}_{37} \mathrm{~N}_{5} \mathrm{O}_{9}(623 \mathrm{~g} / \mathrm{mol})$, m.p.: $184^{\circ} \mathrm{C}$, calc.: C: $59.71 \%$, H: $5.94 \%$, N: $11.24 \%$, found: $\mathrm{C} 59.51 \%$, H: $6.13 \%$, $\mathrm{N}: 11.03 \%,{ }^{1} \mathrm{H}$ NMR $\left(300 \mathrm{MHz}, \mathrm{DMSO}-\mathrm{d}_{6}\right): \delta=11.9(\mathrm{~s}, 1 \mathrm{H}, \mathrm{C}=\mathrm{NNH}), 8.5(\mathrm{~s}, 1 \mathrm{H}, \mathrm{N}=\mathrm{CH}), 8.0-7.2(\mathrm{~m}, 8 \mathrm{H}$, arom. $\underline{\mathrm{H}}), 7.4(\mathrm{~d}, 1 \mathrm{H}, \mathrm{CHNH}), 7.0\left(\mathrm{t}, 1 \mathrm{H}, \mathrm{CH}_{2} \mathrm{NH}\right), 4.3(\mathrm{~m}, 1 \mathrm{H}, \mathrm{C} \underline{\mathrm{H}}), 3.4\left(\mathrm{~m}, 2 \mathrm{H}, \mathrm{CH}_{2}\right), 2.8\left(\mathrm{~s}, 4 \mathrm{H}, \underline{\mathrm{H}}_{2} \mathrm{C}-\mathrm{CH}_{2}\right)$, $1.4 / 1.3\left(2 \mathrm{~s}, 18 \mathrm{H}, 6 \mathrm{CH}_{3}\right),{ }^{\mathrm{T}} \mathrm{C}$ NMR $\left(75.4 \mathrm{MHz}, \mathrm{DMSO}-\mathrm{d}_{6}\right): \delta=177.7\left(\mathrm{OC}_{-} \mathrm{CH}_{2} \mathrm{CH}_{2}-\mathrm{CO}\right), 170.5(\mathrm{CHCO})$, $163.1(\mathrm{C}=\mathrm{NNH}-\mathrm{CO}), 156.5 / 156.2(2 \mathrm{NCOO}), 152.6(\underline{\mathrm{C}}=\mathrm{N}), 148.1 / 135.0 / 134.0 / 133.0 / 131.3 / 129.9 / 129.1 /$ $127.9 / 127.6 / 123.0$ (arom. $\underline{\mathrm{C}}), 79.6 / 79.1\left(2 \underline{\mathrm{C}}\left(\mathrm{CH}_{3}\right)_{3}\right), 55.1(\underline{\mathrm{C}} \mathrm{H}), 41.7\left(\underline{\mathrm{CH}_{2}}\right), 28.4\left(\mathrm{H}_{2} \underline{\mathrm{C}}-\underline{\mathrm{C}} \mathrm{H}_{2}\right), 28.0\left(6 \underline{\mathrm{CH}}_{3}\right)$

4-|N,N'-bis-(tert.-butoxycarbonyl)-D, L-2,3-diaminopropanoyloxy|-acetophenone 3-succinimidobenzoylhydrazone (28)

$5.76 \mathrm{mmol} 26(2 \mathrm{~g})$ and $5.76 \mathrm{mmol} 16(2.43 \mathrm{~g})$ were dissolved in $50 \mathrm{ml}$ THF and stirred for $2 \mathrm{~h}$ at room temperature. THF was then evaporated in vacuo and the residue dissolved in a minimal amount of ethyl acetate and purified by column chromatography (silica gel, ethyl acetate / $\mathrm{n}$-hexane 1:1) to yield $1.20 \mathrm{~g}$ $(1.89 \mathrm{mmol}, 32.8 \%) 28$.

$\mathrm{R}_{\mathrm{f}}=0.23$ (ethyl acetate / n-hexane 1:1), $\mathrm{C}_{32} \mathrm{H}_{39} \mathrm{~N}_{5} \mathrm{O}_{9}\left(637 \mathrm{~g} / \mathrm{mol}\right.$ ), m.p.: $168{ }^{\circ} \mathrm{C}$, calc.: C: $60.28 \%, \mathrm{H}: 6.12 \%$, $\mathrm{N}: 10.99 \%$, found: C: $60.40 \%, \mathrm{H}: 6.15 \%, \mathrm{~N}: 10.70 \%,{ }^{1} \mathrm{H}$ NMR $\left(300 \mathrm{MHz}, \mathrm{DMSO}-\mathrm{d}_{6}\right): \delta=10.9(\mathrm{~s}, 1 \mathrm{H}$, $\mathrm{C}=\mathrm{NNH}), 7.9-7.0(\mathrm{~m}, 8 \mathrm{H}$, arom. $\underline{\mathrm{H}}), 7.4(\mathrm{~d}, 1 \mathrm{H}, \mathrm{CHNH}), 6.9\left(\mathrm{t}, 1 \mathrm{H}, \mathrm{CH}_{2} \mathrm{NH}\right), 4.3(\mathrm{~m}, 1 \mathrm{H}, \mathrm{CH}), 3.4(\mathrm{~m}, 2 \mathrm{H}$, $\left.\mathrm{CH}_{2}\right), 2.8\left(\mathrm{~s}, 4 \mathrm{H}, \underline{\mathrm{H}}_{2} \mathrm{C}-\mathrm{CH}_{2}\right), 2.3\left(\mathrm{~s}, 3 \mathrm{H}, \mathrm{N}=\mathrm{C}-\mathrm{CH}_{3}\right), 1.4 / 1.3\left(2 \mathrm{~s}, 18 \mathrm{H}, 6 \mathrm{C}_{3}\right),{ }^{13} \mathrm{C} \mathrm{NMR}(75.4 \mathrm{MHz}$, DMSO-d $)_{6}: \delta=177.0\left(\overline{\mathrm{OC}}-\mathrm{CH}_{2} \mathrm{CH}_{2}-\mathrm{CO}\right), 169.9(\mathrm{CHCO}), 163.3(\mathrm{C}=\mathrm{NNH}-\mathrm{CO}), 155.8 / 155.6(2 \mathrm{NCOO})$, $151.6(\underline{C}=\mathrm{N}), 135.9 / 135.0 / 133.1 / 132.9 / 130.3 / 128.9 / 127.8 / 127.1 / 121.7$ (arom. $\left.\underline{C}), 78.9 / 78.4\left(2 \mathrm{C}_{\left(\mathrm{CH}_{3}\right.}\right)_{3}\right), 54.4$ $(\underline{\mathrm{C}} \mathrm{H}), 41.1\left(\underline{\mathrm{C}}_{2}\right), 28.7\left(\mathrm{H}_{2} \underline{\mathrm{C}}-\underline{\mathrm{C}} \mathrm{H}_{2}\right), 28.3\left(6 \underline{\mathrm{CH}}_{3}\right), 26.6\left(\mathrm{~N}=\mathrm{C}-\mathrm{CH}_{3}\right)$

\section{Literature}

[1] Heim, M. E. in: Metal Complexes in Cancer Chemotherapy (ed. B. K. Keppler), Verlag Chemie, Weinheim (1993), p. 9

[2] Maeda, H. and Matsumura, Y. Crit. Rev. Ther. Drug Carrier Sys. 6 (1989), 1930

[3] Kratz, F. and Beyer, U. Drug Delivery 5 (1998), 1

[4] Kratz, F., Beyer, U., Roth, T., Tarasova, N., Collery, P., Lechenault, F., Cazabat, A., Schumacher, P., Unger, C. and Falken, U. J. Pharm. Sci. 87 (1998), 338

[5] Kratz, F., Beyer, U., Collery, P., Lechenault, F., Cazabat, A., Schumacher, P., Falken, U. and Unger, C. Biol. Pharm. Bull. 21 (1998), 56

[6] Kratz, F., Beyer, U., Schumacher, P., Krüger, M., Zahn, H., Roth, T., Fiebig, H. H. and Unger, C. Bioorg. Med. Chem. Lett. 7 (1997), 617

[7] Beyer, U., Roth, T., Schumacher, P., Maier, G., Unold, A., Frahm, A. W., Fiebig, H. H., Unger, C. and Kratz, F. J. Med. Chem. 41 (1998), 2701 
[8] Kratz, F., Beyer, U., Roth, T., Schütte, M. T., Unold, A., Fiebig, H. H. and Unger, C. Arch. Pharm. Pharm. Med. Chem. 331 (1998), 47

[9] Drevs, J., Hofmann, I., Marmé, D., Unger, C. and Kratz, F. Drug Delivery 6 (1999), 1

[10] Kratz, F., Beyer, U. and Schütte, M. T. Crit. Rev. Ther. Drug Carrier Sys. 16 (1999), 245

[11] Schütte, M. T., Schumacher, P., Unger, C., Mülhaupt, R. and Kratz, F. Inorg. Chim. Acta 267 (1998), 133

[12] Beyer, U., Krüger, M., Schumacher, P., Unger, C. and Kratz, F. Monatshefte der Chemie 128 (1997), 91

[13] Benassi, R. and Taddei F. J. Chem. Soc., Perkin Trans. II 10 (1985), 1629

[14] Slotta, K. H. and Kethur, R. Chem. Ber. 71 (1938), 335

Received: November 29, 1999 - Accepted: December 17, 1999 Received in revised camera-ready format: March 8, 2000 